\title{
CONVERGENCE OF THE UNIAXIAL PERFECTLY MATCHED LAYER METHOD FOR TIME-HARMONIC SCATTERING PROBLEMS IN TWO-LAYERED MEDIA*
}

\author{
ZHIMING $\mathrm{CHEN}^{\dagger}$ AND WEIYING ZHENG $^{\ddagger}$
}

\begin{abstract}
In this paper, we propose a uniaxial perfectly matched layer (PML) method for solving the time-harmonic scattering problems in two-layered media. The exterior region of the scatterer is divided into two half spaces by an infinite plane, on two sides of which the wave number takes different values. We surround the computational domain where the scattering field is interested by a PML layer with the uniaxial medium property. By imposing homogenous boundary condition on the outer boundary of the PML layer, we show that the solution of the PML problem converges exponentially to the solution of the original scattering problem in the computational domain as either the PML absorbing coefficient or the thickness of the PML layer tends to infinity.
\end{abstract}

1. Introduction. We propose and study the uniaxial perfectly matched layer (PML) method for solving Helmholtz scattering problems in two-layered media:

$$
\begin{aligned}
\Delta u+k^{2} u=f & \text { in } \Omega=\mathbb{R}^{2} \backslash \bar{D}, \\
u=g & \text { on } \Gamma_{D}, \\
(1+r)^{-1 / 2}\left(\frac{\partial u}{\partial r}-\mathbf{i} k u\right) & \in L^{2}\left(\mathbb{R}^{2} \backslash \overline{B\left(R_{0}\right)}\right) .
\end{aligned}
$$

Here $r=|x|$ and $f \in\left(H^{1}(\Omega)\right)^{\prime}$ has the support inside $B\left(R_{0}\right)=\left\{x \in \mathbb{R}^{2}:|x|<R_{0}\right\}$, where $\left(H^{1}(\Omega)\right)^{\prime}$ is the dual space of $H^{1}(\Omega), D \subset \mathbb{R}^{2}$ is a bounded domain with Lipschitz boundary $\Gamma_{D}$, and $g \in H^{1 / 2}\left(\Gamma_{D}\right)$. We assume the wave number $k$ is positive and piecewise constant, defined by

$$
k(x)= \begin{cases}k_{1}, & \text { if } x \in \mathbb{R}_{+}^{2}, \\ k_{2}, & \text { if } x \in \mathbb{R}_{-}^{2},\end{cases}
$$

where $\mathbb{R}_{ \pm}^{2}=\left\{\left(x_{1}, x_{2}\right) \in \mathbb{R}^{2}: \pm x_{2}>0\right\}$. Without loss of generality we assume in this paper that $k_{2}>k_{1}>0$. We remark that the boundary condition (1.2) is not essential for our results. In fact, (1.2) can be replaced by other boundary conditions such as Neumann or impedance boundary conditions on $\Gamma_{D}$. We refer to Coyle and Monk [12] and Monk [18] for finite element methods solving scattering problems in layered media.

The uniqueness of the Helmholtz scattering problems with two unbounded domains is established in Odeh [20] and Kristensson [15] under the following form of Sommerfeld radiation condition

$$
\lim _{R \rightarrow \infty} \int_{\Gamma_{R}}\left|\frac{\partial u}{\partial r}-\mathbf{i} k u\right|^{2} d s=0
$$

where $\Gamma_{R}$ is the circle of radius $R$. (1.3) is another variant of the Sommerfeld radiation condition introduced in Roach and Zhang [22] with which the existence and uniqueness

${ }^{*}$ LSEC, Institute of Computational Mathematics, Academy of Mathematics and System Sciences, Chinese Academy of Sciences, Beijing 100190, People's Republic of China.

$\dagger$ This author was supported in part by China NSF under the grant 10428105 and by the National Basic Research Project under the grant 2005CB321701. (zmchen@lsec.cc.ac.cn).

$\ddagger$ This author was supported in part by China NSF under the grant 60873177 and by the National Basic Research Project under the grant 2005CB321702. (zwy@lsec.cc.ac.cn). 
of solutions to the wave propagation problem with two unbounded media are proved by the method of limiting absorption principle. We shall briefly consider the existence and uniqueness of (1.1)-(1.3) based on the results in [22.

Since the work of Bérénger [2] which proposed a PML method for solving the time dependent Maxwell equations, various constructions of PML absorbing layers have been proposed and studied in the literature (cf. e.g. Hagstrom [13], Turkel and Yefet 25, Teixeira and Chew 24, for the reviews). The basic idea of the PML method is to surround the computational domain by a layer of finite thickness with specially designed model medium that absorbs all the waves that propagate from inside the computational domain.

The convergence of the PML method for homogeneous background materials has drawn considerable attentions in the literature. Lassas and Somersalo 16, 17, Hohage et al 14 studied the acoustic scattering problems for circular and smooth PML layers. It is proved in [14, 16, 17 that the PML solution converges exponentially to the solution of the original scattering problem as the thickness of the PML layer tends to infinity. In the practical application of PML methods, the adaptive PML method was proposed in Chen and $\mathrm{Wu}[4$ for a scattering problem by periodic structures (the grating problem), in Chen and Liu [5] for the acoustic scattering problem, and in Chen and Chen [3] for Maxwell scattering problems. The main idea of the adaptive PML method is to use the a posteriori error estimate to determine the PML parameters and to use the adaptive finite element method to solve the PML equations. The adaptive PML method provides a complete numerical strategy to solve the scattering problems in the framework of finite element which produces automatically a coarse mesh size away from the fixed domain and thus makes the total computational costs insensitive to the thickness of the PML absorbing layer.

The purpose of this paper is to study the convergence of the uniaxial PML (UPML) method for the scattering problem (1.1)-(1.3). The UPML method is widely used in the engineering literatures and has the advantage over the circular PML method in that it provides greater flexibility and efficiency to solve problems involving anisotropic scatterers. The convergence result established in this paper constitutes an important step in studying efficient numerical methods such as adaptive UPML method for solving scattering problems in layered media. In Chen and $\mathrm{Wu}[6$, the adaptive UPML method is proposed for Helmholtz scattering problems with constant wave number.

Since the background materials in the upper and lower half spaces are different, the scattering waves will change their directions at the interface $\Sigma=\left\{\left(x_{1}, 0\right): x_{1} \in \mathbb{R}\right\}$ and split into reflective and refractive waves on two sides of $\Sigma$. The Green function of the scattering problem in layered media becomes very complicated. Our convergence proof is based on the Cagniard de-Hoop transformation of the Green function and the idea of the complex coordinate stretching. By using the integral representation of the exterior Helmholtz equation and some elaborated estimation of the modified Green function, we show that the solution of the UPML problem converges exponentially to the solution of (1.1)-(1.3) as either the PML absorbing coefficient or the thickness of the PML layer tends to infinity.

The layout of the paper is as follows. In section 2 we study the Green function for the scattering problem in the two-layer media. We recall the derivation of the Green function by the method of Fourier transform and derive an alternative form of the Green function which is crucial for the convergence analysis by using the Cagniardde Hoop transformation. In section 3 we prove an integral representation of the 
exterior Helmholtz equation in two-layer media. In section 4 we introduce the UPML formulation for (1.1)-(1.3) by following the method of complex coordinate stretching in Chew and Weedon [6], Collino and Monk [10. In section 5 we study the stability of the Dirichlet problem of UPML equation in the PML layer. In section 6 we study the exponential decay estimate for the modified Green function in the PML layer. In section 7 we prove the convergence of the UPML method.

2. Green function. In this section we study the Green function for the layered media

$$
\Delta_{x} G(x, y)+k^{2} G(x, y)=-\delta_{y}(x) \quad \text { in } \mathbb{R}^{2},
$$

where $\delta_{y}(x)$ is the Dirac source at $y \in \mathbb{R}_{+}^{2}$ or $y \in \mathbb{R}_{-}^{2}$. We will first derive the formula for the Green function by using the method of Fourier transform and the Sommerfeld Integral Path. Next we will use the Cagniard-de Hoop transform to obtain a new formula for the Green function which will be crucial for us to prove the exponential decay of the PML extension in Section 6.

2.1. The method of Fourier transform. We first consider the case $y \in \mathbb{R}_{+}^{2}$, that is, $y_{2}>0$. Let

$$
\hat{G}\left(\xi, x_{2}\right)=\frac{1}{\sqrt{2 \pi}} \int_{-\infty}^{\infty} G_{y}\left(x_{1}, x_{2}\right) e^{-\mathbf{i}\left(x_{1}-y_{1}\right) \xi} \mathrm{d} x_{1}
$$

be the Fourier transform of $G_{y}\left(x_{1}, x_{2}\right)=G(x, y)$ for the first variable. By taking the Fourier transform of (2.1) in the first variable, we obtain

$$
\frac{\partial^{2} \hat{G}}{\partial x_{2}^{2}}+\left(k^{2}-\xi^{2}\right) \hat{G}=-\frac{1}{\sqrt{2 \pi}} \delta_{y_{2}}\left(x_{2}\right)
$$

Throughout the paper we will always assume that for $z \in \mathbb{C}, z^{1 / 2}$ is the analytic branch of $\sqrt{z}$ such that $\operatorname{Re}\left(z^{1 / 2}\right) \geq 0$. This corresponds to the left half real axis as the branch cut in the complex plane. For $z=z_{1}+\mathbf{i} z_{2}, z_{1}, z_{2} \in \mathbb{R}$, we have

$$
z^{1 / 2}=\sqrt{\frac{|z|+z_{1}}{2}}+\operatorname{isgn}\left(z_{2}\right) \sqrt{\frac{|z|-z_{1}}{2}} .
$$

For $z$ on the left half real axis, we take $z^{1 / 2}$ as the limit of $(z+\mathbf{i} \varepsilon)^{1 / 2}$ as $\varepsilon \rightarrow 0^{+}$.

Denote $v_{j}=e^{\mathbf{i} \mu_{j}\left|x_{2}-y_{2}\right|}$, where $\mu_{j}=\left(k_{j}^{2}-\xi^{2}\right)^{1 / 2}, j=1,2$. It is easy to see that

$$
\frac{\partial^{2} v_{j}}{\partial x_{2}^{2}}+\left(k_{j}^{2}-\xi^{2}\right) v_{j}=2 \mathbf{i} \mu_{j} \delta_{y_{2}}\left(x_{2}\right) \text {. }
$$

For $y_{2}>0$, we write the solution of (2.2) as

$$
\hat{G}\left(\xi, x_{2}\right)=\frac{1}{\sqrt{2 \pi}} \begin{cases}\frac{\mathbf{i}}{2 \mu_{1}} e^{\mathbf{i} \mu_{1}\left|x_{2}-y_{2}\right|}+\hat{G}_{1}\left(\xi, x_{2}\right) & \text { if } x_{2}>0 \\ \hat{G}_{2}\left(\xi, x_{2}\right) & \text { if } x_{2}<0 .\end{cases}
$$

Combining (2.2) and (2.4) we find that

$$
\frac{\partial^{2} \hat{G}_{j}}{\partial x_{2}^{2}}+\left(k_{j}^{2}-\xi^{2}\right) \hat{G}_{j}=0,
$$


which has two fundamental solutions $e^{\mathbf{i} \mu_{j} x_{2}}$ and $e^{-\mathbf{i} \mu_{j} x_{2}}, j=1,2$. By allowing only bounded Fourier modes for the Green function, we choose $\hat{G}_{1}=A e^{\mathbf{i} \mu_{1} x_{2}}$ and $\hat{G}_{2}=$ $B e^{-\mathbf{i} \mu_{2} x_{2}}$. By the continuity of $\hat{G}$ and $\frac{\partial \hat{G}}{\partial x_{2}}$ across the interface $\Sigma$, we know that

$$
A=\frac{\mathbf{i}}{2 \mu_{1}} \frac{\mu_{1}-\mu_{2}}{\mu_{1}+\mu_{2}} e^{\mathbf{i} \mu_{1} y_{2}}, \quad B=\frac{\mathbf{i}}{\mu_{1}+\mu_{2}} e^{\mathbf{i} \mu_{1} y_{2}} .
$$

Therefore

$$
\hat{G}\left(\xi, x_{2}\right)=\frac{1}{\sqrt{2 \pi}} \begin{cases}\frac{\mathbf{i}}{2 \mu_{1}} e^{\mathbf{i} \mu_{1}\left|x_{2}-y_{2}\right|}+\frac{\mathbf{i}}{2 \mu_{1}} \frac{\mu_{1}-\mu_{2}}{\mu_{1}+\mu_{2}} e^{\mathbf{i} \mu_{1}\left(x_{2}+y_{2}\right)}, & \text { if } x_{2}>0, \\ \frac{\mathbf{i}}{\mu_{1}+\mu_{2}} e^{\mathbf{i}\left(\mu_{1} y_{2}-\mu_{2} x_{2}\right)}, & \text { if } x_{2}<0 .\end{cases}
$$

The desired Green function should be obtained by taking the inverse Fourier transform of $\hat{G}\left(\xi, x_{2}\right)$. Unfortunately, one cannot simply take the inverse Fourier transform in the above formula because the branch cuts for $\mu_{j}$ are the half lines $\left(-\infty,-k_{j}\right]$ and $\left[k_{j}, \infty\right), j=1,2$, in the complex $\xi$-plane. One way to solve the problem is to take the Sommerfeld Integral Path (SIP) as the integral path for the inverse Fourier transform (see Figure 2.1 for the SIP for the real wave number $k_{1}$ and $k_{2}$ ). From Fig. 2.1] it is clear that $\operatorname{Re} \xi \cdot \operatorname{Im} \xi \leq 0$ for any $\xi \in \mathrm{SIP}$ which indicates that $\operatorname{Im} \mu_{j} \geq 0, j=1,2$. We refer to [9, Chapter 2] for more discussion on the Sommerfeld Integral Paths.

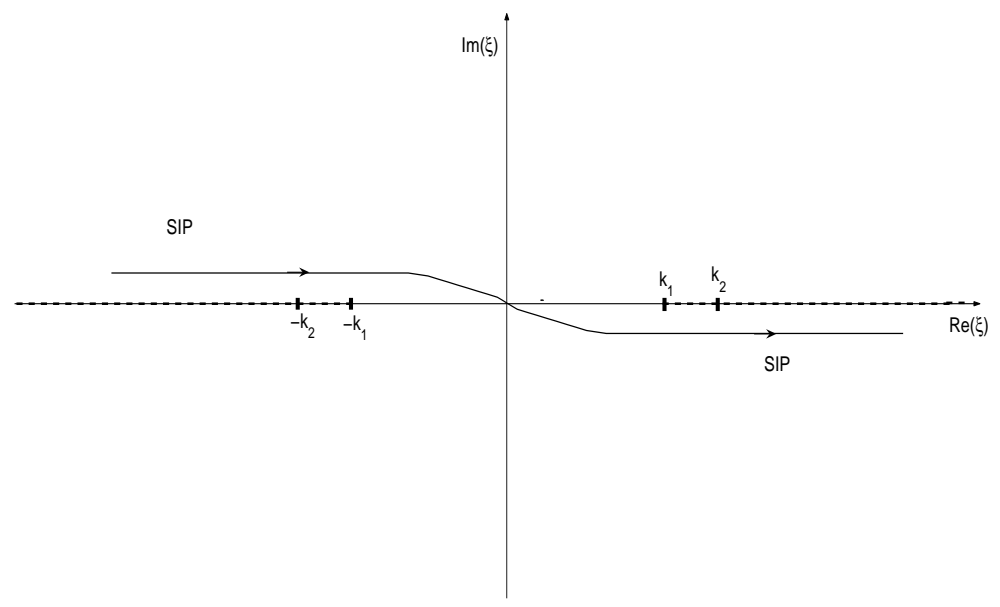

FIG. 2.1. The Sommerfeld Integral Path.

Recall that the Green function for the Helmholtz equation with constant wave number $k_{1}$ is $\Phi\left(k_{1}, x, y\right)=\frac{\mathbf{i}}{4} H_{0}^{(1)}\left(k_{1}|x-y|\right)$ which satisfies (cf. e.g. [9])

$$
\Phi\left(k_{1}, x, y\right)=\frac{\mathbf{i}}{4 \pi} \int_{\operatorname{SIP}} \frac{1}{\mu_{1}} e^{\mathbf{i}\left(x_{1}-y_{1}\right) \xi+\mathbf{i} \mu_{1}\left|x_{2}-y_{2}\right|} \mathrm{d} \xi .
$$

By taking the inverse Fourier transform of $\hat{G}\left(\xi, x_{2}\right)$ using the SIP, we obtain the Green function $G(x, y)$ for $x \in \mathbb{R}_{+}^{2}, y \in \mathbb{R}_{+}^{2}$,

$$
G(x, y)=\Phi\left(k_{1}, x, y\right)-\Phi\left(k_{1}, x, y^{\prime}\right)+\frac{\mathbf{i}}{2 \pi} \int_{\operatorname{SIP}} \frac{1}{\mu_{1}+\mu_{2}} e^{\mathbf{i} \xi\left(x_{1}-y_{1}\right)+\mathbf{i} \mu_{1}\left(x_{2}+y_{2}\right)} \mathrm{d} \xi
$$


where $y^{\prime}=\left(y_{1},-y_{2}\right)$ is the image of $y=\left(y_{1}, y_{2}\right)$, and for $x \in \mathbb{R}_{-}^{2}, y \in \mathbb{R}_{+}^{2}$,

$$
G(x, y)=\frac{\mathbf{i}}{2 \pi} \int_{\operatorname{SIP}} \frac{1}{\mu_{1}+\mu_{2}} e^{\mathbf{i} \xi\left(x_{1}-y_{1}\right)+\mathbf{i}\left(\mu_{1} y_{2}-\mu_{2} x_{2}\right)} \mathrm{d} \xi .
$$

Similarly we can deduce the Green function for $x \in \mathbb{R}_{+}^{2}, y \in \mathbb{R}_{-}^{2}$,

$$
G(x, y)=\frac{\mathbf{i}}{2 \pi} \int_{\operatorname{SIP}} \frac{1}{\mu_{1}+\mu_{2}} e^{\mathbf{i} \xi\left(x_{1}-y_{1}\right)+\mathbf{i}\left(\mu_{1} x_{2}-\mu_{2} y_{2}\right)} \mathrm{d} \xi,
$$

and for $x \in \mathbb{R}_{-}^{2}, y \in \mathbb{R}_{-}^{2}$,

$$
G(x, y)=\Phi\left(k_{2}, x, y\right)-\Phi\left(k_{2}, x, y^{\prime}\right)+\frac{\mathbf{i}}{2 \pi} \int_{\operatorname{SIP}} \frac{1}{\mu_{1}+\mu_{2}} e^{\mathbf{i} \xi\left(x_{1}-y_{1}\right)-\mathbf{i} \mu_{2}\left(x_{2}+y_{2}\right)} \mathrm{d} \xi
$$

In (2.6)-(2.7), the point source is located at $y \in \mathbb{R}_{+}^{2}$. The first term on the righthand side of (2.6) stands for the incident waves coming from the source, and the other terms are the reflected waves by the interface. The righthand side of (2.7) stands for the refractive waves below the interface. Similarly (2.9) represents the combination of incident and reflected waves and (2.8) represents refractive waves when the point source is located at $y \in \mathbb{R}_{-}^{2}$. It is clear that $G(x, y)$ is smooth when $x \neq y$.

2.2. The Cagniard-de Hoop transform. Let $h$ be a bounded analytic function in $\mathbb{C} \backslash\left(\left(-\infty,-k_{1}\right] \cup\left[k_{1}, \infty\right)\right)$. For any $a \in \mathbb{R}, b>0$, we denote

$$
I(h ; a, b)=\frac{\mathbf{i}}{2 \pi} \int_{\text {SIP }} \frac{h(\xi)}{\mu_{1}+\mu_{2}} e^{\mathbf{i} \xi a+\mathbf{i} \mu_{1} b} \mathrm{~d} \xi .
$$

It is easy to see that the Green function $G(x, y)$ can be represented as follows: for $y \in \mathbb{R}_{+}^{2}$,

$$
G(x, y)= \begin{cases}\Phi\left(k_{1}, x, y\right)-\Phi\left(k_{1}, x, y^{\prime}\right)+I\left(1 ; x_{1}-y_{1}, x_{2}+y_{2}\right), & \text { if } x_{2}>0 \\ I\left(e^{\mathbf{i}\left(\mu_{1}-\mu_{2}\right) x_{2}} ; x_{1}-y_{1},-x_{2}+y_{2}\right), & \text { if } x_{2}<0\end{cases}
$$

and for $y \in \mathbb{R}_{-}^{2}$,

$$
G(x, y)= \begin{cases}I\left(e^{\mathbf{i}\left(\mu_{1}-\mu_{2}\right) y_{2}}, x_{1}-y_{1}, x_{2}-y_{2}\right), & \text { if } x_{2}>0 \\ \Phi\left(k_{2}, x, y\right)-\Phi\left(k_{2}, x, y^{\prime}\right) & \\ +I\left(e^{\mathbf{i}\left(\mu_{1}-\mu_{2}\right)\left(x_{2}+y_{2}\right)} ; x_{1}-y_{1},-x_{2}-y_{2}\right), & \text { if } x_{2}<0\end{cases}
$$

LEMma 2.1. Let $a \in \mathbb{R}, b>0, \rho=\sqrt{a^{2}+b^{2}}$, and $h$ be a bounded analytic function in $\mathbb{C} \backslash\left(\left(-\infty,-k_{1}\right] \cup\left[k_{1}, \infty\right)\right)$ satisfying $h(\xi)=h(-\xi)$ and $\overline{h(\xi)}=h(\bar{\xi})$. Then

$$
I(h ; a, b)=\frac{1}{\pi} \int_{1}^{\infty} \frac{1}{\sqrt{t^{2}-1}} \operatorname{Re}\left[\left(\frac{\mu_{1}}{\mu_{1}+\mu_{2}} h\right)(\xi)\right] e^{\mathrm{i} k_{1} \rho t} \mathrm{~d} t,
$$

where $\xi=\frac{k_{1}|a| t}{\rho}+\mathbf{i} \frac{k_{1} b \sqrt{t^{2}-1}}{\rho}$ and $\mu_{j}=\left(k_{j}^{2}-\xi^{2}\right)^{1 / 2}, j=1,2$.

Proof. Notice that the Sommerfeld Integral Path is symmetric with respect to the origin of the complex $\xi$-plane (see Figure 2.1). From (2.10) and $h(\xi)=h(-\xi)$ we find that

$$
I(h ; a, b)=I(h ;-a, b)=\frac{\mathbf{i}}{2 \pi} \int_{\operatorname{SIP}} \frac{h(\xi)}{\mu_{1}+\mu_{2}} e^{\mathbf{i} \xi|a|+\mathbf{i} \mu_{1} b} \mathrm{~d} \xi .
$$


Without loss of generality we assume $a \geq 0$ in the rest of the proof.

We use the method of Cagniard-de Hoop transform to prove the lemma. Let $t^{\prime}=\xi a+\mu_{1} b$. Then it is easy to see that

$$
\xi=\frac{k_{1} a t}{\rho} \pm \mathbf{i} \frac{k_{1} b \sqrt{t^{2}-1}}{\rho}, \quad t=\frac{t^{\prime}}{k_{1} \rho} .
$$

Let $\xi=\xi_{1}+\mathbf{i} \xi_{2}, \xi_{1}, \xi_{2} \in \mathbb{R}$, then

$$
b^{2} \xi_{1}^{2}-a^{2} \xi_{2}^{2}=k_{1}^{2} a^{2} b^{2} / \rho^{2} .
$$

For $t \in[1, \infty), \xi(t)$ is the right branch of hyperbola that intersects the real axis at $\xi_{0}=k_{1} a / \rho$. It is easy to see that

$$
k_{1}^{2}-\xi^{2}=\left(\frac{k_{1} b t}{\rho} \mp \mathbf{i} \frac{k_{1} a}{\rho} \sqrt{t^{2}-1}\right)^{2} .
$$

Since $\operatorname{Re}\left(\mu_{1}\right) \geq 0$, we have, for $t \in[1, \infty)$,

$$
\mu_{1}=\frac{k_{1} b t}{\rho} \mp \mathrm{i} \frac{k_{1} a}{\rho} \sqrt{t^{2}-1},
$$

and consequently $\frac{\mathrm{d} \xi}{\mathrm{d} t}= \pm \mathbf{i} \frac{\mu_{1}}{\sqrt{t^{2}-1}}$.

Let $\Gamma_{+}, \Gamma_{-}$be respectively the parts of the hyperbola in the upper-half complex plane and the lower-half complex plane. For any $r>0$, denote $C_{r}^{+}, C_{r}^{-}$be respectively the part of the circle $\{\xi:|\xi|=r\}$ that are bounded by the SIP and $\Gamma_{+}$or by the SIP and $\Gamma_{-}$. The geometry is depicted in Figure 2.2.

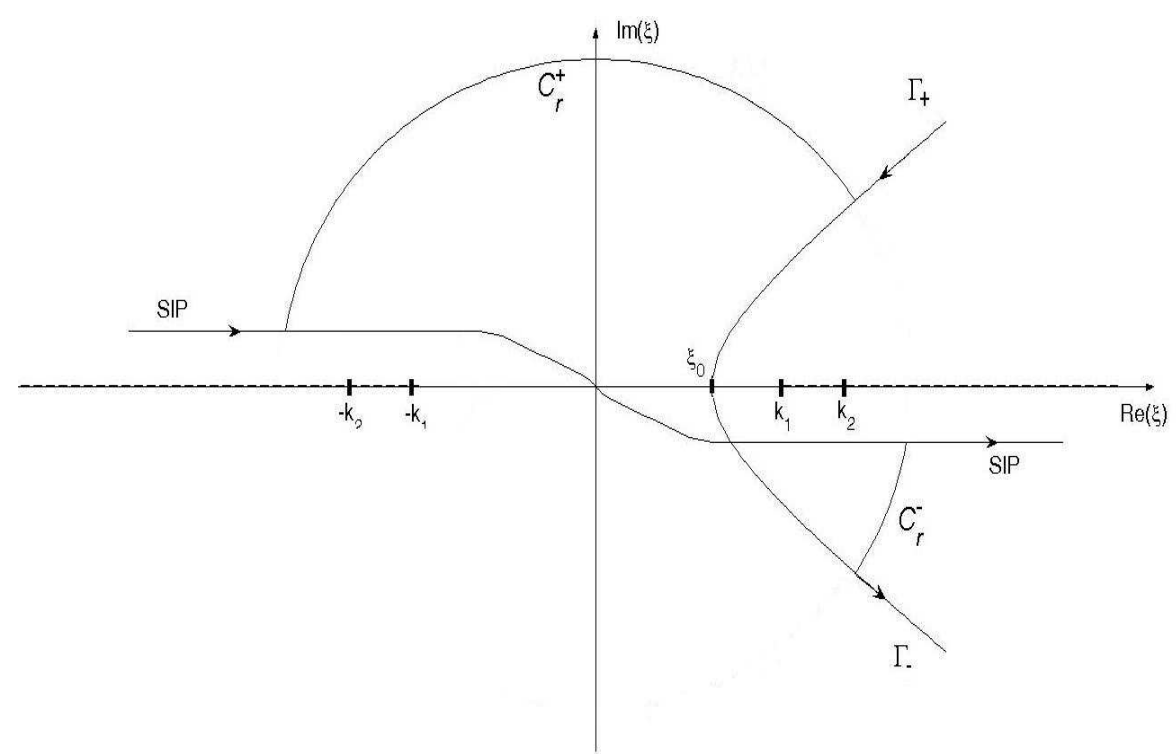

FIG. 2.2. Cagniard-de Hoop transform from the SIP to $\Gamma_{+} \cup \Gamma_{-}$.

For the integrals on $C_{r}^{ \pm}$we have

$$
\lim _{r \rightarrow \infty}\left|\int_{C_{r}^{ \pm}} \frac{h}{\mu_{1}+\mu_{2}} e^{\mathbf{i} \xi a+\mathbf{i} \mu_{1} b} \mathrm{~d} \xi\right|=0 .
$$


We will postpone the proof of (2.16) at the end of the proof of this lemma. Now notice that for $b>0, \xi_{0}<k_{1}$, by using Cauchy integral theorem and letting $r \rightarrow \infty$, we obtain

$$
I(h ; a, b)=\frac{\mathbf{i}}{2 \pi} \int_{\text {SIP }} \frac{h}{\mu_{1}+\mu_{2}} e^{\mathbf{i} \xi a+\mathbf{i} \mu_{1} b} \mathrm{~d} \xi=\frac{\mathbf{i}}{2 \pi} \int_{\Gamma_{+} \cup \Gamma_{-}} \frac{h}{\mu_{1}+\mu_{2}} e^{\mathbf{i} \xi a+\mathbf{i} \mu_{1} b} \mathrm{~d} \xi,
$$

which by the transform (2.13) yields

$$
I(h ; a, b)=\frac{1}{2 \pi} \int_{1}^{\infty} \frac{1}{\sqrt{t^{2}-1}}\left[\left(\frac{\mu_{1}}{\mu_{1}+\mu_{2}} h\right)\left(\xi_{+}\right)+\left(\frac{\mu_{1}}{\mu_{1}+\mu_{2}} h\right)\left(\xi_{-}\right)\right] e^{\mathbf{i} k_{1} \rho t} \mathrm{~d} t .
$$

This implies the desired formula by using the fact that $\xi_{+}=\xi, \xi_{-}=\bar{\xi}$, and $h(\bar{\xi})=$ $\overline{h(\xi)}$.

Now we prove (2.16). Let $\xi=r e^{\mathbf{i} \theta}$. By the convention in (2.3), it is easy to check that for $r>k_{2}>k_{1}$,

$$
\left|\mu_{1}+\mu_{2}\right| \geq \frac{\sqrt{2}}{2}\left(\sqrt{r^{2}-k_{1}^{2}}+\sqrt{r^{2}-k_{2}^{2}}\right) .
$$

Since $h$ is bounded in $\mathbb{C} \backslash\left(\left(-\infty,-k_{1}\right] \cup\left[k_{1}, \infty\right)\right)$ whose upper bound is denoted as $M(h)$, we obtain

$$
\begin{aligned}
\left|\int_{C_{r}^{+}} \frac{h}{\mu_{1}+\mu_{2}} e^{\mathrm{i} \xi a+\mathbf{i} \mu_{1} b} \mathrm{~d} \xi\right| & \leq\left|\int_{\theta_{1}}^{\theta_{2}} \frac{|h(\xi)|}{\left|\mu_{1}(\xi)+\mu_{2}(\xi)\right|} e^{-\operatorname{Im}\left(\xi a+\mu_{1}(\xi) b\right)} r \mathrm{~d} \theta\right| \\
& \leq \sqrt{2} M(h) \int_{\theta_{1}}^{\theta_{2}} \frac{r}{\sqrt{r^{2}-k_{1}^{2}}+{\sqrt{r^{2}-k_{2}^{2}}}^{-\operatorname{Im}\left(\xi a+\mu_{1} b\right)} \mathrm{d} \theta}
\end{aligned}
$$

where $\xi_{2}=r e^{\mathbf{i} \theta_{2}}$ and $\xi_{1}=r e^{\mathbf{i} \theta_{1}}$ are respectively the intersection point of $C_{r}^{+}$with SIP and $\Gamma_{+}$. We have $0<\theta_{1}<\theta_{2}<\pi$.

If $\theta \in\left(\pi / 2, \theta_{2}\right)$, we have $\operatorname{Im}\left(\mu_{1}^{2}\right)=\operatorname{Im}\left(-r^{2} \sin 2 \theta\right)>0$ and thus

$$
\operatorname{Im}\left(\xi a+\mu_{1} b\right) \geq \operatorname{ar} \sin \theta>\operatorname{ar} \sin \theta_{2} .
$$

If $\theta \in\left(\theta_{1}, \pi / 2\right)$, we have $\operatorname{Im}\left(\mu_{1}^{2}\right)=\operatorname{Im}\left(-r^{2} \sin 2 \theta\right)<0$ and consequently

$$
\operatorname{Im}\left(\xi a+\mu_{1} b\right)=a r \sin \theta-\left[\frac{\left|k_{1}^{2}-r^{2} e^{2 \mathbf{i} \theta}\right|-\left(k_{1}^{2}-r^{2} \cos 2 \theta\right)}{2}\right]^{1 / 2} b,
$$

which is an increasing function in $\left[\theta_{1}, \pi / 2\right]$ for $r^{2} \geq 2 k_{1}^{2}$. Thus, for $\xi=r e^{\mathbf{i} \theta}, \theta_{1} \leq \theta \leq$ $\pi / 2$,

$$
\operatorname{Im}\left(\xi a+\mu_{1} b\right) \geq \operatorname{Im}\left(\xi_{1} a+\mu_{1}\left(\xi_{1}\right) b\right)=k_{1} \rho t_{1},
$$

where $t_{1} \in(1, \infty)$ satisfies $\xi_{1}=r e^{\mathbf{i} \theta_{1}}=\frac{k_{1} a t_{1}}{\rho}+\mathbf{i} \frac{k_{1} b \sqrt{t_{1}^{2}-1}}{\rho}$. It is clear that $t_{1} \rightarrow \infty$ as $r \rightarrow \infty$. Therefore

$$
\begin{aligned}
\left|\int_{C_{r}^{+}} \frac{h}{\mu_{1}+\mu_{2}} e^{\mathrm{i} \xi a+\mathbf{i} \mu_{1} b} \mathrm{~d} \xi\right| \leq & \sqrt{2} M(h) \int_{\pi / 2}^{\theta_{2}} \frac{r}{\sqrt{r^{2}-k_{1}^{2}}+\sqrt{r^{2}-k_{2}^{2}}} e^{-a r \sin \theta_{2}} \mathrm{~d} \theta \\
& +\sqrt{2} M(h) \int_{\theta_{1}}^{\pi / 2} \frac{r}{\sqrt{r^{2}-k_{1}^{2}}+\sqrt{r^{2}-k_{2}^{2}}} e^{-k_{1} \rho t_{1}} \mathrm{~d} \theta \\
\rightarrow & 0, \quad \text { as } r \rightarrow \infty .
\end{aligned}
$$


This shows (2.16) for the integral on $C_{r}^{+}$. The proof for the integral on $C_{r}^{-}$is similar and we omit the details. This completes the proof.

The new representation of the Green function based on the Cagniard-de Hoop transform is useful in the convergence analysis of the PML method where the complex continuation of the Green function plays a decisive role. In the literature for the efficient computation of Green functions (cf. e.g. Paulus et al 21]), on the other hand, one deforms the integral path such that the integrand decays exponentially on the new integral path. Then the integral can be computed numerically over small intervals.

3. The scattering problem. We start with the following uniqueness result which can be proved by the method in Roach and Zhang [22, Theorem 3.1], [23, Theorem 3.1].

Lemma 3.1. The scattering problem (1.1) - (1.3) has at most one solution $u \in$ $H_{\mathrm{loc}}^{1}(\Omega)$.

The existence of the solution to (1.1) - (1.3) can be proved by the method of limiting absorption principle as in 22. Here we briefly recall the argument. For any $z=\lambda+\mathbf{i} \tau \in \mathbb{C}, \lambda>0, \tau>0$, and $f_{1} \in\left(H^{1}\left(\mathbb{R}^{2}\right)\right)^{\prime}$, we consider the following problem

$$
\Delta u_{z}+z k^{2}(x) u_{z}=f_{1} \quad \text { in } \mathbb{R}^{2} .
$$

It is easy to see by Lax-Milgram lemma that (3.1) has a unique solution $u_{z} \in H^{1}\left(\mathbb{R}^{2}\right)$ when $\tau>0$. For any domain $U \subset \mathbb{R}^{2}$, we define the weighted space $L^{2, \varepsilon}(U), \varepsilon \in \mathbb{R}$, by

$$
L^{2, \varepsilon}(U)=\left\{v \in L_{\mathrm{loc}}^{2}(U):(1+|x|)^{\varepsilon} v \in L^{2}(U)\right\}
$$

with the weighted norm $\|v\|_{\varepsilon, U}=\left(\int_{\Omega}(1+|x|)^{2 \varepsilon}|v|^{2} \mathrm{~d} x\right)^{1 / 2}$. We also need the space $B^{*}(U)$ which is a subspace of $L_{\mathrm{loc}}^{2}(U)$ such that

$$
\|v\|_{B^{*}(U)}^{2}=\sup _{R>1} \frac{1}{R} \int_{U \cap B(R)}|v(x)|^{2} \mathrm{~d} x<+\infty \quad \forall v \in B^{*}(U) .
$$

The following key result in proving the existence is established in 22, Theorem 3.2 and (3.18)].

Lemma 3.2. Let $f_{1} \in L^{2,(3+\delta) / 2}\left(\mathbb{R}^{2}\right), 0<\delta<1$, and $z=\lambda+\mathbf{i} \tau, 0<\lambda_{0}<\lambda<\lambda_{1}$, $0<\tau \leq 1$. Let $\frac{1}{2}-\frac{1}{4} \delta<\sigma<1$. Then

$$
\left\|\partial_{r} u_{z}-\mathbf{i} z^{1 / 2} k u_{z}\right\|_{-\sigma / 2, \mathbb{R}^{2}}+\left\|u_{z}\right\|_{B^{*}\left(\mathbb{R}^{2}\right)} \leq C\left\|f_{1}\right\|_{(3+\delta) / 2, \mathbb{R}^{2}}
$$

for some fixed constant $C>0$, independent of $\tau, u_{z}, f_{1}$.

THEOREM 3.3. For any $f \in\left(H^{1}(\Omega)\right)^{\prime}$ with the support included in $B\left(R_{0}\right)$ and $g \in H^{1 / 2}\left(\Gamma_{D}\right)$, the scattering problem (1.1) - (1.3) has a unique solution $u \in H_{\mathrm{loc}}^{1}(\Omega)$ that satisfies

$$
\|u\|_{B^{*}(\Omega)} \leq C\left(\|f\|_{\left(H^{1}(\Omega)\right)^{\prime}}+\|g\|_{H^{1 / 2}\left(\Gamma_{D}\right)}\right) .
$$

Proof. By Lemma 3.1 we only need to prove the existence. For any $\tau \in(0,1)$, we consider the following problem

$$
\begin{aligned}
\Delta w_{\tau}+(1+\mathbf{i} \tau) k^{2}(x) w_{\tau}=f & \text { in } \Omega, \\
w_{\tau}=g & \text { on } \Gamma_{D} . \\
8 &
\end{aligned}
$$


It is easy to show by Lax-Milgram lemma that (3.3)-(3.4) has a unique solution $w_{\tau} \in H^{1}(\Omega)$. Let $\chi \in C_{0}^{\infty}\left(\mathbb{R}^{2}\right)$ be the cut-off function such that $0 \leq \chi \leq 1, \chi=0$ in $B\left(R_{0}\right)$, and $\chi=1$ outside $B\left(R_{0}+1\right)$. Let $v_{\tau}=\chi w_{\tau}$. Then $v_{\tau}$ satisfies (3.1) with $z=1+\mathbf{i} \tau$ and $f_{1}=\chi f+\Delta \chi w_{\tau}+2 \nabla \chi \cdot \nabla w_{\tau}$. It is clear that $f_{1}$ has a compact support and $\left\|f_{1}\right\|_{(3+\delta) / 2, \mathbb{R}^{2}} \leq C\left\|w_{\tau}\right\|_{H^{1}\left(\Omega\left(R_{0}+1\right)\right)}$ for some constant $C>0$ depending only on $R_{0}$. Here $\Omega\left(R_{0}+1\right)=\Omega \cap B\left(R_{0}+1\right)$. By Lemma 3.2 we know that

$$
\left\|\partial_{r} v_{\tau}-\mathbf{i}(1+\mathbf{i} \tau)^{1 / 2} k v_{\tau}\right\|_{-\sigma / 2, \mathbb{R}^{2}}+\left\|v_{\tau}\right\|_{B^{*}\left(\mathbb{R}^{2}\right)} \leq C\left\|w_{\tau}\right\|_{H^{1}\left(\Omega\left(R_{0}+1\right)\right)} .
$$

This implies

$$
\left\|\partial_{r} w_{\tau}-\mathbf{i}(1+\mathbf{i} \tau)^{1 / 2} k w_{\tau}\right\|_{-\sigma / 2, E\left(R_{0}+1\right)}+\left\|w_{\tau}\right\|_{B^{*}(\Omega)} \leq C\left\|w_{\tau}\right\|_{H^{1}\left(\Omega\left(R_{0}+1\right)\right)},
$$

where $E\left(R_{0}+1\right)=\mathbb{R}^{2} \backslash \overline{B\left(R_{0}+1\right)}$. Now let $\chi_{1} \in C_{0}^{\infty}\left(\mathbb{R}^{2}\right)$ be the cut-off function such that $0 \leq \chi_{1} \leq 1, \chi_{1}=1$ in $B\left(R_{0}+1\right), \chi_{1}=0$ outside $B\left(R_{0}+2\right)$. Denote $w_{g} \in H^{1}(\Omega)$ as the lifting of the function $g \in H^{1 / 2}\left(\Gamma_{D}\right)$ such that $w_{g}=g$ on $\Gamma_{D}$ and $\left\|w_{g}\right\|_{H^{1}(\Omega)} \leq C\|g\|_{H^{1 / 2}\left(\Gamma_{D}\right)}$. By multiplying (3.3) with $\chi_{1}^{2}\left(\overline{w_{\tau}-w_{g}}\right)$ and using the standard argument we have

$$
\left\|w_{\tau}\right\|_{H^{1}\left(\Omega\left(R_{0}+1\right)\right)} \leq C\left(\|f\|_{\left(H^{1}(\Omega)\right)^{\prime}}+\|g\|_{H^{1 / 2}\left(\Gamma_{D}\right)}+\left\|w_{\tau}\right\|_{L^{2}\left(\Omega\left(R_{0}+2\right)\right)}\right) .
$$

Now we claim that

$$
\left\|w_{\tau}\right\|_{L^{2}\left(\Omega\left(R_{0}+2\right)\right)} \leq C\left(\|f\|_{\left(H^{1}(\Omega)\right)^{\prime}}+\|g\|_{H^{1 / 2}\left(\Gamma_{D}\right)}\right)
$$

for any $f \in\left(H^{1}(\Omega)\right)^{\prime}$ with the support inside $B\left(R_{0}\right), g \in H^{1 / 2}\left(\Gamma_{D}\right)$, and $\tau \in(0,1)$. The argument is similar to that in [22. If (3.7) were false, then there would be sequences $\left\{f_{m}\right\} \subset\left(H^{1}(\Omega)\right)^{\prime}$ with the support of $f_{m}$ inside $B\left(R_{0}\right),\left\{g_{m}\right\} \subset H^{1 / 2}\left(\Gamma_{D}\right)$, $\left\{\tau_{m}\right\} \subset(0,1)$, and $\left\{w_{\tau_{m}}\right\}$ the corresponding solution of (3.3)-(3.4) such that

$$
\left\|w_{\tau_{m}}\right\|_{L^{2}\left(\Omega\left(R_{0}+2\right)\right)}=1 \text { and }\left\|f_{m}\right\|_{\left(H^{1}(\Omega)\right)^{\prime}}+\left\|g_{m}\right\|_{H^{1 / 2}\left(\Gamma_{D}\right)} \leq 1 / m .
$$

There is a subsequence of $\left\{\tau_{m}\right\}$, which is still denoted by $\left\{\tau_{m}\right\}$, such that $\tau_{m} \rightarrow \tau \in$ $[0,1]$. It follows from the Rellich selection theorem and standard elliptic estimates that there is a subsequence of $\left\{w_{\tau_{m}}\right\}$, which is still denoted by $\left\{w_{\tau_{m}}\right\}$, such that $w_{\tau_{m}}$ converges weakly to some $w \in H_{\text {loc }}^{1}(\Omega)$ which satisfies

$$
\left\|\partial_{r} w-\mathbf{i}(1+\mathbf{i} \tau)^{1 / 2} k w\right\|_{-\sigma / 2, E\left(R_{0}+1\right)} \leq C .
$$

This implies that $w$ satisfies (3.3)-(3.4) with $f=0$ and $g=0$. If $\tau>0$, the uniqueness of the solution in $H^{1}(\Omega)$ indicates $w=0$. If $\tau=0$, we know from (3.9) that $w$ satisfies the radiation condition (1.3) and conclude $w=0$ by Lemma 3.1. However, this contradicts to (3.8). Therefore, we have (3.7) and consequently

$$
\left\|\partial_{r} w_{\tau}-\mathbf{i}(1+\mathbf{i} \tau)^{1 / 2} k w_{\tau}\right\|_{-\sigma / 2, E\left(R_{0}+1\right)}+\left\|w_{\tau}\right\|_{B^{*}(\Omega)} \leq C\left(\|f\|_{\left(H^{1}(\Omega)\right)^{\prime}}+\|g\|_{H^{1 / 2}\left(\Gamma_{D}\right)}\right) .
$$

Now it is easy to see that $w_{\tau}$ has a convergent subsequence which converges weakly to some $w$ in $H_{\mathrm{loc}}^{1}(\Omega)$ that satisfies (1.1)-(1.3). The desired estimate (3.2) follows from the above inequality since $L^{2,-\sigma / 2}\left(E\left(R_{0}+1\right)\right) \subset L^{2,-1 / 2}\left(E\left(R_{0}+1\right)\right)$ for $0<\sigma<1$. This completes the proof. $\square$

The Green function $G(x, y)$ introduced in Section 2.1 based on the Sommerfeld Integral Paths is in fact the consequence of the method of limiting absorption principle 
[9. Chapter 2]. More precisely, for any $z=\lambda+\mathbf{i} \tau \in \mathbb{C}, \lambda>0, \tau>0$, let $G_{z}(x, y)$ be the Green function

$$
\Delta_{x} G_{z}(x, y)+z k^{2}(x) G_{z}(x, y)=-\delta_{y}(x) \quad \text { in } \mathbb{R}^{2} .
$$

Then $G(x, y)$ is the limit of $G_{z}(x, y)$ as $\lambda=1, \tau \rightarrow 0$ for any $x, y \in \mathbb{R}_{ \pm}^{2}, x \neq y$. By Lemma 3.2 and the argument in the proof of Theorem 3.3 we can easily obtain

$$
\left\|\partial_{r} G(\cdot, y)-\mathbf{i} k G(\cdot, y)\right\|_{-\sigma / 2, E\left(R_{1}\right)} \leq C\|G(\cdot, y)\|_{H^{1 / 2}\left(\Gamma_{R_{1}}\right)},
$$

where $\sigma<1$ is defined in Lemma 3.2, $E\left(R_{1}\right)=\mathbb{R}^{2} \backslash \bar{B}\left(R_{1}\right)$, and $B\left(R_{1}\right)$ is any fixed circle that includes $y$. Therefore, for any fixed $y \in \mathbb{R}_{ \pm}^{2}$, the Green function $G(x, y)$ satisfies the radiation condition (1.3).

Our next goal is to show the integral representation of the solution of the exterior Dirichlet problem which plays an important role in our subsequent analysis for the PML problem. The proof of the following lemma uses classical arguments (see e.g. [11, Theorem 3.3, Page 10]) and is included here for the sake of completeness.

Let $D$ and the support of $f$ be contained in the rectangle $B_{1}=\left\{x \in \mathbb{R}^{2}:\left|x_{1}\right|<\right.$ $\left.L_{1} / 2,\left|x_{2}\right|<L_{2} / 2\right\}$. Let $\Gamma_{1}=\partial B_{1}$ and $\mathbf{n}_{1}$ the unit outer normal to $\Gamma_{1}$.

Lemma 3.4. Any solution $u$ of the exterior Dirichlet problem (1.1)-(1.3) satisfies

$$
u(x)=-\Psi_{\mathrm{SL}}(\lambda)(x)+\Psi_{\mathrm{DL}}(g)(x) \quad \text { in } \Omega \backslash \bar{B}_{1},
$$

where $\lambda=\partial u / \partial \mathbf{n}_{1} \in H^{-1 / 2}\left(\Gamma_{1}\right)$ is the Neumann trace of $u$ on $\Gamma_{1}, \mathbf{n}_{1}$ is the unit outer normal to $\Gamma_{1}$, and $\Psi_{\mathrm{SL}}, \Psi_{\mathrm{DL}}$ are respectively the single and double layer potentials

$$
\begin{aligned}
& \Psi_{\mathrm{SL}}(\lambda)(x)=\int_{\Gamma_{1}} G(x, y) \lambda(y) \mathrm{d} s(y), \quad \forall \lambda \in H^{-1 / 2}\left(\Gamma_{1}\right), \\
& \Psi_{\mathrm{DL}}(g)(x)=\int_{\Gamma_{1}} \frac{\partial G(x, y)}{\partial \mathbf{n}_{1}(y)} g(y) \mathrm{d} s(y), \quad \forall g \in H^{1 / 2}\left(\Gamma_{1}\right) .
\end{aligned}
$$

Proof. For any $R>0$ sufficiently large such that $\bar{B}_{1} \subset B(R)$, by the third Green formula, we know that, for any $x \in B(R) \backslash \bar{B}_{1}$,

$$
u(x)=\int_{\Gamma_{1} \cup \Gamma_{R}}\left[\frac{\partial u(y)}{\partial \mathbf{n}(y)} G(x, y)-\frac{\partial G(x, y)}{\partial \mathbf{n}(y)} u(y)\right] \mathrm{d} s(y),
$$

where $\Gamma_{R}=\partial B(R)$ and $\mathbf{n}$ is the unit outer normal to the boundary of $B(R) \backslash \bar{B}_{1}$. By the radiation condition (1.3), we know that

$$
\liminf _{R \rightarrow \infty} \int_{\Gamma_{R}}\left[\left|\frac{\partial u}{\partial r}\right|^{2}+k^{2}|u|^{2}+2 k \operatorname{Im}\left(u \frac{\partial \bar{u}}{\partial r}\right)\right] \mathrm{d} s=\liminf _{R \rightarrow \infty} \int_{\Gamma_{R}}\left|\frac{\partial u}{\partial r}-\mathbf{i} k u\right|^{2} \mathrm{~d} s=0 .
$$

Thus there exists a sequence $R_{j} \rightarrow \infty$ such that

$$
\lim _{R_{j} \rightarrow \infty} \int_{\Gamma_{R_{j}}}\left[\left|\frac{\partial u}{\partial r}\right|^{2}+k^{2}|u|^{2}+2 k \operatorname{Im}\left(u \frac{\partial \bar{u}}{\partial r}\right)\right] \mathrm{d} s=0 .
$$

On the other hand, since $\bar{D}$ and the support of $f$ are contained in $B\left(R_{0}\right)$, again by the third Green formula we know that for $R_{j}>R_{0}$,

$$
\int_{\Gamma_{R_{j}}} \operatorname{Im}\left(u \frac{\partial \bar{u}}{\partial r}\right) \mathrm{d} s=\int_{\Gamma_{R_{0}}} \operatorname{Im}\left(u \frac{\partial \bar{u}}{\partial r}\right) \mathrm{d} s<\infty .
$$


Thus we know from (3.14) that $\|u\|_{L^{2}\left(\Gamma_{R_{j}}\right)}$ is bounded as $R_{j} \rightarrow \infty$. Similarly, since $G(\cdot, y)$ satisfies the radiation condition (1.3), without loss of generality, we may assume that $\|G(\cdot, y)\|_{L^{2}\left(\Gamma_{R_{j}}\right)}$ is also bounded as $R_{j} \rightarrow \infty$ and $\left\|\partial_{r} G(\cdot, y)-\mathbf{i} k G(\cdot, y)\right\|_{L^{2}\left(R_{j}\right)} \rightarrow 0$ as $R_{j} \rightarrow \infty$. Now the lemma follows from (3.13), (3.14), and the fact that

$$
\begin{aligned}
& \int_{\Gamma_{R_{j}}}\left[\frac{\partial u(y)}{\partial \mathbf{n}(y)} G(x, y)-\frac{\partial G(x, y)}{\partial \mathbf{n}(y)} u(y)\right] \mathrm{d} s(y) \\
= & \int_{\Gamma_{R_{j}}}\left[\left(\frac{\partial u(y)}{\partial \mathbf{n}(y)}-\mathbf{i} k u(y)\right) G(x, y)-\left(\frac{\partial G(x, y)}{\partial \mathbf{n}(y)}-\mathbf{i} k G(x, y)\right) u(y)\right] \mathrm{d} s(y) \\
\rightarrow & 0 \quad \text { as } R_{j} \rightarrow \infty .
\end{aligned}
$$

This completes the proof.

We conclude this section by introducing the equivalent weak formulation of the original scattering problem (1.1)-(1.3). We start by introducing the Dirichlet-toNeumann operator $T: H^{1 / 2}\left(\Gamma_{1}\right) \rightarrow H^{-1 / 2}\left(\Gamma_{1}\right)$. Given $\zeta \in H^{1 / 2}\left(\Gamma_{1}\right)$, we define $T \zeta=\frac{\partial \chi}{\partial \mathbf{n}_{1}}$ on $\Gamma_{1}$, where $\chi$ is the solution of the following exterior Dirichlet problem of the Helmholtz equation

$$
\begin{aligned}
& \Delta \chi+k^{2}(x) \chi=0 \quad \text { in } \mathbb{R}^{2} \backslash \bar{B}_{1}, \\
& \chi=\zeta \quad \text { on } \Gamma_{1}, \quad(1+r)^{-1 / 2}\left(\frac{\partial \chi}{\partial r}-\mathbf{i} k \chi\right) \in L^{2}\left(E\left(R_{1}\right)\right),
\end{aligned}
$$

where $k(x)$ is piecewise constant defined in (1.4), $E\left(R_{1}\right)=\mathbb{R}^{2} \backslash \overline{B\left(R_{1}\right)}$ with $R_{1}>0$ sufficiently large such that $\bar{B}_{1} \subset B\left(R_{1}\right)$. By Theorem 3.3, $T: H^{1 / 2}\left(\Gamma_{1}\right) \rightarrow H^{-1 / 2}\left(\Gamma_{1}\right)$ is well-defined and is a continuous linear operator.

Let $a: H^{1}\left(\Omega_{1}\right) \times H^{1}\left(\Omega_{1}\right) \rightarrow \mathbb{C}$, where $\Omega_{1}=B_{1} \backslash \bar{D}$, be the sesquilinear form

$$
a(\varphi, \psi)=\int_{\Omega_{1}}\left(\nabla \varphi \cdot \nabla \bar{\psi}-k^{2} \varphi \bar{\psi}\right) \mathrm{d} x-\langle T \varphi, \psi\rangle_{\Gamma_{1}},
$$

where $\langle\cdot, \cdot\rangle_{\Gamma_{1}}$ stands for the inner product on $L^{2}\left(\Gamma_{1}\right)$ or the duality pairing between $H^{-1 / 2}\left(\Gamma_{1}\right)$ and $H^{1 / 2}\left(\Gamma_{1}\right)$. The scattering problem (1.1)-(1.3) is equivalent to the following weak formulation: Given $f \in\left(H^{1}(\Omega)\right)^{\prime}$ with the support included in $\Omega_{1}$ and $g \in H^{1 / 2}\left(\Gamma_{D}\right)$, find $u \in H^{1}\left(\Omega_{1}\right)$ such that $u=g$ on $\Gamma_{D}$ and

$$
a(u, \psi)=(f, \psi)_{\Omega_{1}}, \quad \forall \psi \in H_{\Gamma_{D}}^{1}\left(\Omega_{1}\right),
$$

where $(\cdot, \cdot)_{\Omega_{1}}$ is the inner product on $L^{2}\left(\Omega_{1}\right)$ or the duality pairing between $\left(H^{1}\left(\Omega_{1}\right)\right)^{\prime}$ and $H^{1}\left(\Omega_{1}\right), H_{\Gamma_{D}}^{1}\left(\Omega_{1}\right)=\left\{v \in H^{1}\left(\Omega_{1}\right): v=0\right.$ on $\left.\Gamma_{D}\right\}$.

For the sesquilinear form $a(\cdot, \cdot)$ we associate with a bounded linear operator $\hat{A}$ : $H_{\Gamma_{D}}^{1}\left(\Omega_{1}\right) \rightarrow\left(H_{\Gamma_{D}}^{1}\left(\Omega_{1}\right)\right)^{\prime}$ such that

$$
(\hat{A} \varphi, \psi)_{\Omega_{1}}=a(\varphi, \psi), \quad \forall \varphi, \psi \in H_{\Gamma_{D}}^{1}\left(\Omega_{1}\right) .
$$

By Theorem $3.3 \hat{A}$ is surjective and one-to-one. Hence we know from the open mapping theorem that $\hat{A}^{-1}$ is bounded, which implies that there exists a constant $C>0$ such that the following inf-sup condition is satisfied

$$
\sup _{0 \neq \psi \in H_{\Gamma_{D}}^{1}\left(\Omega_{1}\right)} \frac{|a(\varphi, \psi)|}{\|\psi\|_{H^{1}\left(\Omega_{1}\right)}} \geq C\|\varphi\|_{H^{1}\left(\Omega_{1}\right)}, \quad \forall \varphi \in H_{\Gamma_{D}}^{1}\left(\Omega_{1}\right) .
$$


4. The uniaxial PML equation. Now we introduce the absorbing PML layer. Let $B_{2}=\left\{x \in \mathbb{R}^{2}:\left|x_{1}\right|<L_{1} / 2+d_{1},\left|x_{2}\right|<L_{2} / 2+d_{2}\right\}$ be the rectangle which contains $B_{1}$. Let $\alpha_{1}(t)=1+\mathbf{i} \sigma_{1}(t), \alpha_{2}(t)=1+\mathbf{i} \sigma_{2}(t)$ be the model medium property which satisfy

$$
\sigma_{j} \geq 0, \quad \sigma_{j}(t)=\sigma_{j}(-t), \quad \text { and } \sigma_{j}=0 \text { for }|t| \leq L_{j} / 2, \quad j=1,2 .
$$

Denote by $\tilde{x}_{j}$ the complex coordinate defined by

$$
\tilde{x}_{j}=\int_{0}^{x_{j}} \alpha_{j}(t) \mathrm{d} t, \quad j=1,2 .
$$

Notice that $\tilde{x}_{j}$ depends only on $x_{j}$ and for this reason the method is called the uniaxial PML method. The complex distance is defined by

$$
\rho(\tilde{x}, y)=\left[\left(\tilde{x}_{1}-y_{1}\right)^{2}+\left(\tilde{x}_{2}-y_{2}\right)^{2}\right]^{1 / 2} .
$$

We follow the method of complex coordinate stretching [8, 4, to introduce the PML equation. We define

$$
\tilde{G}(x, y):=G(\tilde{x}, y), \quad \forall x, y \in \mathbb{R}^{2} .
$$

From (2.11)-(2.12) and (4.1), it is easy to see that $\tilde{G}(\cdot, y) \in C\left(\mathbb{R}^{2} \backslash\{y\}\right)$ and $\tilde{G}(x, \cdot) \in$ $C^{1}\left(\mathbb{R}^{2} \backslash\{x\}\right)$. Now we can define the modified single and double layer potentials

$$
\begin{aligned}
& \tilde{\Psi}_{\mathrm{SL}}(\lambda)(x)=\int_{\Gamma_{1}} \tilde{G}(x, y) \lambda(y) \mathrm{d} s(y), \quad \forall \lambda \in H^{-1 / 2}\left(\Gamma_{1}\right), \\
& \tilde{\Psi}_{\mathrm{DL}}(\zeta)(x)=\int_{\Gamma_{1}} \frac{\partial \tilde{G}(x, y)}{\partial \mathbf{n}_{1}(y)} \zeta(y) \mathrm{d} s(y), \quad \forall \zeta \in H^{1 / 2}\left(\Gamma_{1}\right) .
\end{aligned}
$$

It is clear that $\tilde{\Psi}_{\mathrm{SL}}(\lambda), \tilde{\Psi}_{\mathrm{DL}}(\zeta)$ are continuous in $\mathbb{R}^{2} \backslash \bar{B}_{1}$, and

$$
\begin{array}{ll}
\gamma_{D}^{+} \tilde{\Psi}_{\mathrm{SL}}(\lambda)=\gamma_{D}^{+} \Psi_{\mathrm{SL}}(\lambda), & \forall \lambda \in H^{-1 / 2}\left(\Gamma_{1}\right), \\
\gamma_{D}^{+} \tilde{\Psi}_{\mathrm{DL}}(\zeta)=\gamma_{D}^{+} \Psi_{\mathrm{DL}}(\zeta), & \forall \zeta \in H^{1 / 2}\left(\Gamma_{1}\right),
\end{array}
$$

where $\gamma_{D}^{+}: H_{\text {loc }}^{1}\left(\mathbb{R}^{2} \backslash \bar{B}_{1}\right) \rightarrow H^{1 / 2}\left(\Gamma_{1}\right)$ is the trace operator.

For any $\zeta \in H^{1 / 2}\left(\Gamma_{1}\right)$, let $\mathbb{E}(\zeta)(x)$ be the PML extension given by

$$
\mathbb{E}(\zeta)(x)=-\tilde{\Psi}_{\mathrm{SL}}(T \zeta)(x)+\tilde{\Psi}_{\mathrm{DL}}(\zeta)(x) \quad \text { for } x \in \mathbb{R}^{2} \backslash \bar{B}_{1} .
$$

By (4.4) and (3.16) we know that, for any $\zeta \in H^{1 / 2}\left(\Gamma_{1}\right)$,

$$
\gamma_{D}^{+} \mathbb{E}(\zeta)=-\gamma_{D}^{+} \Psi_{\mathrm{SL}}(T \zeta)+\gamma_{D}^{+} \Psi_{\mathrm{DL}}(\zeta)=\gamma_{D}^{+} \chi=\zeta \quad \text { on } \Gamma_{1} .
$$

For the solution $u$ of the scattering problem (3.18), let $\tilde{u}=\mathbb{E}\left(\left.u\right|_{\Gamma_{1}}\right)$ be the PML extension of $\left.u\right|_{\Gamma_{1}}$ which satisfies $\gamma_{D}^{+} \tilde{u}=\left.u\right|_{\Gamma_{1}}$ on $\Gamma_{1}$. It is obvious that $\tilde{u}$ satisfies

$$
\frac{\partial^{2} \tilde{u}}{\partial \tilde{x}_{1}^{2}}+\frac{\partial^{2} \tilde{u}}{\partial \tilde{x}_{2}^{2}}+k^{2} \tilde{u}=0 \text { in } \mathbb{R}^{2} \backslash \bar{B}_{1}
$$

which yields the desired UPML equation by the chain rule

$$
\nabla \cdot(A \nabla \tilde{u})+\alpha_{1} \alpha_{2} k^{2} \tilde{u}=0 \quad \text { in } \mathbb{R}^{2} \backslash \bar{B}_{1},
$$


where $A=\operatorname{diag}\left(\alpha_{2}\left(x_{2}\right) / \alpha_{1}\left(x_{1}\right), \alpha_{1}\left(x_{1}\right) / \alpha_{2}\left(x_{2}\right)\right)$ is a diagonal matrix. The UPML solution $\hat{u}$ in $\Omega_{2}=B_{2} \backslash \bar{D}$ is defined as the solution of the following system

$$
\begin{aligned}
& \nabla \cdot(A \nabla \hat{u})+\alpha_{1} \alpha_{2} k^{2} \hat{u}=f \quad \text { in } \Omega_{2}, \\
& \hat{u}=g \quad \text { on } \Gamma_{D}, \quad \hat{u}=0 \quad \text { on } \Gamma_{2} \text {. }
\end{aligned}
$$

The well-posedness of the UPML problem (4.6)-(4.7) and the convergence of the solution $\hat{u}$ to the solution of the original scattering problem will be studied in next sections.

5. The PML equation in the PML layer. In this section we consider the Dirichlet problem of the PML equation in the layer:

$$
\begin{array}{ll}
\nabla \cdot(A \nabla w)+\alpha_{1} \alpha_{2} k^{2} w=0 & \text { in } \Omega_{\mathrm{PML}}=B_{2} \backslash \bar{B}_{1}, \\
w=0 \quad \text { on } \Gamma_{1}, \quad w=q & \text { on } \Gamma_{2},
\end{array}
$$

where $q \in H^{1 / 2}\left(\Gamma_{2}\right)$. Introduce the sesquilinear form $c: H^{1}\left(\Omega_{\mathrm{PML}}\right) \times H^{1}\left(\Omega_{\mathrm{PML}}\right) \rightarrow \mathbb{C}$ as

$$
c(\varphi, \psi)=\int_{\Omega_{\mathrm{PML}}}\left(A \nabla \varphi \cdot \nabla \bar{\psi}-\alpha_{1} \alpha_{2} k^{2} \varphi \bar{\psi}\right) \mathrm{d} x, \quad \forall \varphi, \psi \in H_{0}^{1}\left(\Omega_{\mathrm{PML}}\right) .
$$

Then the weak formulation of (5.1) - (5.2) is: Find $w \in H^{1}\left(\Omega_{\mathrm{PML}}\right)$ such that $w=0$ on $\Gamma_{1}, w=q$ on $\Gamma_{2}$, and

$$
c(w, v)=0, \quad \forall v \in H_{0}^{1}\left(\Omega_{\mathrm{PML}}\right) .
$$

Notice that, for any $\varphi \in H^{1}\left(\Omega_{\mathrm{PML}}\right)$,

$$
\operatorname{Re}[c(\varphi, \varphi)]=\int_{\Omega_{\mathrm{PML}}}\left[\frac{1+\sigma_{1} \sigma_{2}}{1+\sigma_{1}^{2}}\left|\frac{\partial \varphi}{\partial x_{1}}\right|^{2}+\frac{1+\sigma_{1} \sigma_{2}}{1+\sigma_{2}^{2}}\left|\frac{\partial \varphi}{\partial x_{2}}\right|^{2}+k^{2}\left(\sigma_{1} \sigma_{2}-1\right)|\varphi|^{2}\right] \mathrm{d} x .
$$

Since

$$
\frac{1+\sigma_{1} \sigma_{2}}{1+\sigma_{1}^{2}} \geq \frac{1}{1+\sigma_{m}^{2}}, \quad \frac{1+\sigma_{1} \sigma_{2}}{1+\sigma_{2}^{2}} \geq \frac{1}{1+\sigma_{m}^{2}},
$$

where $\sigma_{m}=\max _{x \in \Omega_{\mathrm{PML}}}\left(\sigma_{1}\left(x_{1}\right), \sigma_{2}\left(x_{2}\right)\right)>0$, we know by using the spectral theory of compact operators that for any given $k_{2}$, (5.3) has a unique solution for every real $k_{1}$ except possibly for a discrete set of values of $k_{1}$ (see Collino and Monk [10, Theorem 2] for a similar discussion on the PML equation in the polar coordinates). In this section we will make the following assumption on the medium property

(H1) $\sigma_{j}(t) \equiv \sigma>0, \quad \forall|t| \geq L_{j} / 2, \quad j=1,2$, where $\sigma$ is a positive constant.

This assumption which allows us to prove the coercivity of the sesquilinear form $c$ is not very restrictive in practical applications. In particular, our numerical experiences with the adaptive uniaxial PML for Maxwell scattering problems 7 ] indicate that the constant medium property leads to better preconditioning techniques for the discrete PML problems as opposed to the continuous medium properties.

Throughout the rest of the paper we will use the weighted $H^{1}$-norm

$$
\|\varphi\|_{H^{1}(U)}=\left(\|\nabla \varphi\|_{L^{2}(U)}^{2}+\|k \varphi\|_{L^{2}(U)}^{2}\right)^{1 / 2},
$$


for any bounded domain $U \subset \mathbb{R}^{2}$. For any $\varphi \in H^{1}\left(\Omega_{\mathrm{PML}}\right)$, we define an equivalent norm on $H^{1}\left(\Omega_{\mathrm{PML}}\right)$ by

$$
\|\varphi\|_{*, \Omega_{\mathrm{PML}}}=\left(\|A \nabla \varphi\|_{L^{2}\left(\Omega_{\mathrm{PML})}\right.}^{2}+\left\|k \alpha_{1} \alpha_{2} \varphi\right\|_{L^{2}\left(\Omega_{\mathrm{PML})}\right.}^{2}\right)^{1 / 2} .
$$

Lemma 5.1. Let (H1) be satisfied. Then (5.3) has a unique solution and it holds that

$$
\sup _{0 \neq \psi \in H_{0}^{1}\left(\Omega_{\mathrm{PML}}\right)} \frac{|c(\varphi, \psi)|}{\|\psi \mid\|_{H^{1}\left(\Omega_{\mathrm{PML}}\right)}} \geq \hat{C}\|\varphi\|_{*, \Omega_{\mathrm{PML}}}, \quad \forall \varphi \in H_{0}^{1}\left(\Omega_{\mathrm{PML}}\right)
$$

where

$$
\hat{C}=\frac{\min \left(1, \sigma^{3}\right)}{2\left(1+\sigma^{2}\right)^{2} \max \left(1, k_{2}^{2} d^{2}\right)}, \quad d=\max \left(d_{1}, d_{2}\right) .
$$

Proof. It is clear that $\bar{\Omega}_{\mathrm{PML}}=\bar{\Omega}_{c} \cup \bar{\Omega}_{1} \cup \bar{\Omega}_{2}$, where

$$
\begin{aligned}
& \Omega_{c}=\left\{x \in \Omega_{\mathrm{PML}}:\left|x_{1}\right|>L_{1} / 2,\left|x_{2}\right|>L_{2} / 2\right\}, \\
& \Omega_{1}=\left\{x \in \Omega_{\mathrm{PML}}:\left|x_{1}\right|>L_{1} / 2,\left|x_{2}\right|<L_{2} / 2\right\}, \\
& \Omega_{2}=\left\{x \in \Omega_{\mathrm{PML}}:\left|x_{2}\right|>L_{2} / 2,\left|x_{1}\right|<L_{1} / 2\right\} .
\end{aligned}
$$

Since $\sigma_{1}=\sigma_{2}=\sigma$ in $\Omega_{c}, \sigma_{2}=0$ in $\Omega_{1}$, and $\sigma_{1}=0$ in $\Omega_{2}$, it is east to check that

$$
\begin{aligned}
\frac{1}{\sigma} \operatorname{Im}[c(\varphi, \varphi)]= & \left\|\frac{\partial \varphi}{\partial x_{2}}\right\|_{L^{2}\left(\Omega_{1}\right)}^{2}+\left\|\frac{\partial \varphi}{\partial x_{1}}\right\|_{L^{2}\left(\Omega_{2}\right)}^{2}-\frac{1}{1+\sigma^{2}} \sum_{j=1}^{2}\left\|\frac{\partial \varphi}{\partial x_{j}}\right\|_{L^{2}\left(\Omega_{j}\right)}^{2} \\
& -2\|k \varphi\|_{L^{2}\left(\Omega_{c}\right)}^{2}-\|k \varphi\|_{L^{2}\left(\Omega_{1} \cup \Omega_{2}\right)}^{2} .
\end{aligned}
$$

The key observation is that

$$
\begin{aligned}
& \operatorname{Re}[c(\varphi, \varphi)]-\frac{1}{\sigma} \operatorname{Im}[c(\varphi, \varphi)] \\
= & \|\nabla \varphi\|_{L^{2}\left(\Omega_{c}\right)}^{2}+\left(1+\sigma^{2}\right)\|k \varphi\|_{L^{2}\left(\Omega_{c}\right)}^{2}+\frac{2}{1+\sigma^{2}} \sum_{j=1}^{2}\left\|\frac{\partial \varphi}{\partial x_{j}}\right\|_{L^{2}\left(\Omega_{j}\right)}^{2} .
\end{aligned}
$$

Therefore, for any $\gamma>0$,

$$
\begin{aligned}
& \operatorname{Re}[c(\varphi, \varphi)]+\frac{\gamma-1}{\sigma} \operatorname{Im}[c(\varphi, \varphi)] \\
= & \|\nabla \varphi\|_{L^{2}\left(\Omega_{c}\right)}^{2}+\gamma\left\|\frac{\partial \varphi}{\partial x_{2}}\right\|_{L^{2}\left(\Omega_{1}\right)}^{2}+\gamma\left\|\frac{\partial \varphi}{\partial x_{1}}\right\|_{L^{2}\left(\Omega_{2}\right)}^{2}+\frac{2-\gamma}{1+\sigma^{2}} \sum_{j=1}^{2}\left\|\frac{\partial \varphi}{\partial x_{j}}\right\|_{L^{2}\left(\Omega_{j}\right)}^{2} \\
& +\left(1+\sigma^{2}-2 \gamma\right)\|\varphi\|_{L^{2}\left(\Omega_{c}\right)}^{2}-\gamma\|k \varphi\|_{L^{2}\left(\Omega_{1} \cup \Omega_{2}\right)}^{2} .
\end{aligned}
$$

Since $\varphi=0$ on $\Gamma_{2}$, we deduce easily that

$$
\|\varphi\|_{L^{2}\left(\Omega_{1}\right)}^{2} \leq d_{1}^{2}\left\|\frac{\partial \varphi}{\partial x_{1}}\right\|_{L^{2}\left(\Omega_{1}\right)}^{2}, \quad\|\varphi\|_{L^{2}\left(\Omega_{2}\right)}^{2} \leq d_{2}^{2}\left\|\frac{\partial \varphi}{\partial x_{2}}\right\|_{L^{2}\left(\Omega_{2}\right)}^{2},
$$


which implies

$$
\begin{aligned}
-\gamma\|k \varphi\|_{L^{2}\left(\Omega_{1} \cup \Omega_{2}\right)}^{2} & =\gamma\|k \varphi\|_{L^{2}\left(\Omega_{1} \cup \Omega_{2}\right)}^{2}-2 \gamma\|k \varphi\|_{L^{2}\left(\Omega_{1} \cup \Omega_{2}\right)}^{2} \\
& \geq \gamma\|k \varphi\|_{L^{2}\left(\Omega_{1} \cup \Omega_{2}\right)}^{2}-2 \gamma k_{2}^{2} d^{2} \sum_{j=1}^{2}\left\|\frac{\partial \varphi}{\partial x_{j}}\right\|_{L^{2}\left(\Omega_{j}\right)}^{2},
\end{aligned}
$$

where $d=\max \left(d_{1}, d_{2}\right)$. Substitute the above estimate into (5.5) we obtain

$$
\begin{aligned}
& \operatorname{Re}[c(\varphi, \varphi)]+(\gamma-1) \sigma^{-1} \operatorname{Im}[c(\varphi, \varphi)] \\
\geq & \|\nabla \varphi\|_{L^{2}\left(\Omega_{c}\right)}^{2}+\gamma\left\|\frac{\partial \varphi}{\partial x_{2}}\right\|_{L^{2}\left(\Omega_{1}\right)}^{2}+\gamma\left\|\frac{\partial \varphi}{\partial x_{1}}\right\|_{L^{2}\left(\Omega_{2}\right)}^{2} \\
& +\left(\frac{2-\gamma}{1+\sigma^{2}}-2 \gamma k_{2}^{2} d^{2}\right) \sum_{j=1}^{2}\left\|\frac{\partial \varphi}{\partial x_{j}}\right\|_{L^{2}\left(\Omega_{j}\right)}^{2} \\
& +\left(1+\sigma^{2}-2 \gamma\right)\|\varphi\|_{L^{2}\left(\Omega_{c}\right)}^{2}+\gamma\|k \varphi\|_{L^{2}\left(\Omega_{1} \cup \Omega_{2}\right)}^{2} .
\end{aligned}
$$

Now we take $\gamma=1 /\left(2 \max \left(1, k_{2}^{2} d^{2}\right)\left(1+\sigma^{2}\right)\right)$, then

$$
\frac{2-\gamma}{1+\sigma^{2}}-2 \gamma k_{2}^{2} d^{2} \geq \frac{1}{2\left(1+\sigma^{2}\right)} \geq \gamma, \quad 1+\sigma^{2}-2 \gamma \geq \sigma^{2}
$$

Thus

$$
\begin{aligned}
\max \left(1, \sigma^{-1}\right)|c(\varphi, \varphi)| & \geq \operatorname{Re}[c(\varphi, \varphi)]+(\gamma-1) \sigma^{-1} \operatorname{Im}[c(\varphi, \varphi)] \\
& \geq \min \left(\gamma, \sigma^{2}\right)\|\varphi\|_{H^{1}\left(\Omega_{\mathrm{PML}}\right)}^{2}
\end{aligned}
$$

Since $\min \left(\gamma, \sigma^{2}\right) / \max \left(1, \sigma^{-1}\right) \geq \gamma \min \left(1, \sigma^{3}\right)$, we have

$$
\begin{aligned}
\sup _{0 \neq \psi \in H_{0}^{1}\left(\Omega_{\mathrm{PML}}\right)} \frac{|c(\varphi, \psi)|}{\|\psi \mid\|_{H^{1}\left(\Omega_{\mathrm{PML}}\right)}} & \geq \frac{|c(\varphi, \varphi)|}{\|\varphi\|_{H^{1}\left(\Omega_{\mathrm{PML}}\right)}} \geq \gamma \min \left(1, \sigma^{3}\right)\|\varphi\|_{H^{1}\left(\Omega_{\mathrm{PML}}\right)} \\
& \geq \gamma \frac{\min \left(1, \sigma^{3}\right)}{1+\sigma^{2}}\|\varphi\|_{*, \Omega_{\mathrm{PML}}} .
\end{aligned}
$$

This completes the proof. $\square$

LEMma 5.2. Let (H1) be satisfied and $w$ be the solution of the PML equation in the layer (5.1) -(5.2). Then, for any $v \in H^{1}\left(\Omega_{\mathrm{PML}}\right)$ such that $v=0$ on $\Gamma_{1}$ and $v=q$ on $\Gamma_{2}$,

$$
\left\|\frac{\partial w}{\partial \mathbf{n}}\right\|_{H^{-1 / 2}\left(\Gamma_{1}\right)} \leq\left(1+\hat{C}^{-1}\right)\|v\|_{*, \Omega_{\mathrm{PML}}}
$$

Proof. Since $w-v \in H_{0}^{1}\left(\Omega_{\mathrm{PML}}\right)$, by (5.4) we have

$$
\begin{aligned}
\hat{C}\|w-v\|_{*, \Omega_{\mathrm{PML}}} & \leq \sup _{\varphi \in H_{0}^{1}\left(\Omega_{\mathrm{PML}}\right)} \frac{|c(w-v, \varphi)|}{\|\varphi \mid\|_{H^{1}\left(\Omega_{\mathrm{PML}}\right)}} \\
& =\sup _{\varphi \in H_{0}^{1}\left(\Omega_{\mathrm{PML}}\right)} \frac{|c(v, \varphi)|}{\|\varphi\|_{H^{1}\left(\Omega_{\mathrm{PML}}\right)}} \leq\|v\|_{*, \Omega_{\mathrm{PML}}},
\end{aligned}
$$

where we have used (5.3) and the fact that $|c(v, \varphi)| \leq\|v\|_{*, \Omega_{\mathrm{PML}}} \mid\|\varphi\|_{H^{1}\left(\Omega_{\mathrm{PML}}\right)}$. This proves $\|w\|_{*, \Omega_{\mathrm{PML}}} \leq\left(1+\hat{C}^{-1}\right)\|v\|_{*, \Omega_{\mathrm{PML}}}$. 
To show (5.6), we test (5.1) with any $\varphi \in H^{1}\left(\Omega_{\mathrm{PML}}\right)$ such that $\varphi=0$ on $\Gamma_{2}$ and obtain

$$
\left|\left\langle\frac{\partial w}{\partial \mathbf{n}}, \varphi\right\rangle_{\Gamma_{1}}\right|=|c(w, \varphi)| \leq\|w\|_{*, \Omega_{\mathrm{PML}}}\|\varphi\|_{H^{1}\left(\Omega_{\mathrm{PML}}\right)} .
$$

This completes the proof. $\square$

6. Estimation of the modified Green function. The convergence analysis for the UPML problem depends crucially on the exponential decay estimate for the modified Green's function $\tilde{G}(x, y)$ which is the goal of this section. We start with the following assumption on the fictitious medium property, which is rather mild in the practical application of the UPML method.

$$
\int_{0}^{\frac{L_{1}}{2}+d_{1}} \sigma_{1}(t) \mathrm{d} t=\int_{0}^{\frac{L_{2}}{2}+d_{2}} \sigma_{2}(t) \mathrm{d} t=: \bar{\sigma}, \quad \bar{\sigma}>0 \text { is a constant. }
$$

We also remark that the result of this section does not depend on the assumption of constant medium property (H1). The following elementary lemma is from 6, Lemma 3.2].

Lemma 6.1. For any $z_{1}=a_{1}+\mathbf{i} b_{1}$ and $z_{2}=a_{2}+\mathbf{i} b_{2}$ with $a_{1}, b_{1}, a_{2}, b_{2} \in \mathbb{R}$ such that $a_{1} b_{1}+a_{2} b_{2}>0$ and $a_{1}^{2}+a_{2}^{2}>0$, we have

$$
\operatorname{Im}\left(z_{1}^{2}+z_{2}^{2}\right)^{1 / 2} \geq \frac{a_{1} b_{1}+a_{2} b_{2}}{\sqrt{a_{1}^{2}+a_{2}^{2}}} .
$$

Proof. Because the proof is short, we rewrite it here for the completeness. Since $\operatorname{Im}\left(z_{1}^{2}+z_{2}^{2}\right)=2\left(a_{1} b_{1}+a_{2} b_{2}\right)>0$, by (2.3) we know that

$$
\operatorname{Re}(a+\mathbf{i} b)^{1 / 2}=\sqrt{\frac{\sqrt{a^{2}+b^{2}}+a}{2}}, \quad \operatorname{Im}(a+\mathbf{i} b)^{1 / 2}=\sqrt{\frac{\sqrt{a^{2}+b^{2}}-a}{2}} .
$$

It is easy to check that $\operatorname{Im}(a+\mathbf{i} b)^{1 / 2}$ is a decreasing function in $a \in \mathbb{R}$. Let $z_{1}^{2}+z_{2}^{2}=$ $a+\mathbf{i} b$, then

$$
a+\mathbf{i} b=\left(\sqrt{a_{1}^{2}+a_{2}^{2}}+\mathbf{i} \frac{a_{1} b_{1}+a_{2} b_{2}}{\sqrt{a_{1}^{2}+a_{2}^{2}}}\right)^{2}-\frac{\left(a_{2} b_{1}-a_{1} b_{2}\right)^{2}}{a_{1}^{2}+a_{2}^{2}} .
$$

Let $a^{\prime}=a+\frac{\left(a_{2} b_{1}-a_{1} b_{2}\right)^{2}}{a_{1}^{2}+a_{2}^{2}}$. Since $a_{1} b_{1}+a_{2} b_{2} \geq 0$, we have

$$
\operatorname{Im}\left(a^{\prime}+\mathbf{i} b\right)^{1 / 2}=\frac{a_{1} b_{1}+a_{2} b_{2}}{\sqrt{a_{1}^{2}+a_{2}^{2}}} .
$$

On the other hand, since $a^{\prime}>a$, we know that $\operatorname{Im}(a+\mathbf{i} b)^{1 / 2} \geq \operatorname{Im}\left(a^{\prime}+\mathbf{i} b\right)^{1 / 2}$. This completes the proof.

The following lemma is the complex counterpart of (2.13) and (2.15).

Lemma 6.2. For any $z_{1}=a_{1}+\mathbf{i} b_{1}$ and $z_{2}=a_{2}+\mathbf{i} b_{2}$ with $a_{1}, b_{1}, a_{2}, b_{2} \in \mathbb{R}$ such that $a_{1}, a_{2}, b_{1}, b_{2} \geq 0$, define

$$
\xi=\frac{k_{1}}{\rho}\left(z_{1} t+\mathbf{i} z_{2} \sqrt{t^{2}-1}\right), \quad \rho=\left(z_{1}^{2}+z_{2}^{2}\right)^{1 / 2}, \quad \forall t \in[1, \infty) .
$$


Then $\mu_{1}(\xi)=\left(k_{1}^{2}-\xi^{2}\right)^{1 / 2}$ satisfies

$$
\mu_{1}=\frac{k_{1}}{\rho}\left(z_{2} t-\mathbf{i} z_{1} \sqrt{t^{2}-1}\right), \quad \forall t \in[1, \infty) .
$$

Proof. For any $t \in[1, \infty)$, let $\mu=k_{1}\left(z_{2} t-\mathbf{i} z_{1} \sqrt{t^{2}-1}\right) / \rho$. Clearly $\mu^{2}=k_{1}^{2}-\xi^{2}=$ $\mu_{1}^{2}$. By the convention in (2.3), the lemma follows from $\operatorname{Re}(\mu) \geq 0$ which can be proved by direct calculations. Here we omit the details.

For $\xi$ given in (6.2). Let $\xi=\xi_{1}+\mathbf{i} \xi_{2}$ with $\xi_{1}, \xi_{2} \in \mathbb{R}$. It is easy to check that

$$
\begin{aligned}
& \xi_{1} \xi_{2}=k_{1}^{2} \frac{\left(a_{1} a_{2}+b_{1} b_{2}\right)\left(\left|z_{1}\right|^{2}+\left|z_{2}\right|^{2}\right)}{|\rho|^{4}} f_{1}(t), \quad f_{1}(t):=t t^{\prime}-\beta\left(t^{2}+t^{\prime 2}\right), \\
& \xi_{1}^{2}-\xi_{2}^{2}=\frac{1}{2} k_{1}^{2}+\frac{1}{2} k_{1}^{2} \frac{\left|z_{1}\right|^{4}-\left|z_{2}\right|^{4}}{|\rho|^{4}} f_{2}(t), \quad f_{2}(t):=t^{2}+t^{\prime 2}-4 \beta t t^{\prime},
\end{aligned}
$$

where $t^{\prime}=\sqrt{t^{2}-1}$ and

$$
\beta:=\frac{a_{1} b_{2}-a_{2} b_{1}}{\left|z_{1}\right|^{2}+\left|z_{2}\right|^{2}}
$$

Consequently, for $\rho=\left(z_{1}^{2}+z_{2}^{2}\right)^{1 / 2}$,

$$
|\rho|^{4}=\left|z_{1}\right|^{4}+\left|z_{2}\right|^{4}+2\left(a_{1} a_{2}+b_{1} b_{2}\right)^{2}-2\left(a_{2} b_{1}-a_{1} b_{2}\right)^{2} .
$$

It is easy to check that $\mu_{2}(\xi)=\left(k_{2}^{2}-\xi^{2}\right)^{1 / 2}$ satisfies

$$
\left|\mu_{2}\right|^{4}=4\left(k_{2}^{4}-k_{2}^{2} k_{1}^{2}\right) \frac{\left(a_{1} a_{2}+b_{1} b_{2}\right)^{2}}{|\rho|^{4}}+\frac{k_{1}^{4}}{4} \frac{\left(\left|z_{1}\right|^{2}+\left|z_{2}\right|^{2}\right)^{2}}{|\rho|^{4}}\left(f_{2}(t)-M\right)^{2},
$$

where

$$
M:=\frac{2 k_{2}^{2}-k_{1}^{2}}{k_{1}^{2}} \frac{\left|z_{1}\right|^{2}-\left|z_{2}\right|^{2}}{\left|z_{1}\right|^{2}+\left|z_{2}\right|^{2}}
$$

LEMMA 6.3. For any $z_{1}=a_{1}+\mathbf{i} b_{1}$ and $z_{2}=a_{2}+\mathbf{i} b_{2}$ with $a_{1}, b_{1}, a_{2}, b_{2} \in \mathbb{R}$ such that $a_{1}, a_{2}, b_{1}, b_{2} \geq 0$, define $\rho=\left(z_{1}^{2}+z_{2}^{2}\right)^{1 / 2}$. Assume that

$$
b_{1}^{2}+b_{2}^{2} \geq a_{1}^{2}+a_{2}^{2} .
$$

Then for any $\mu_{j}(\xi)=\left(k_{j}^{2}-\xi^{2}\right)^{1 / 2}$ with $\xi$ given in (6.2), $j=1,2$, we have

$$
\operatorname{Im}\left[\left(\mu_{1}-\mu_{2}\right) z_{2}\right] \leq 0 .
$$

Proof. Denote $\mu_{j}=p_{j}+\mathbf{i} q_{j}$ with $p_{j}, q_{j} \in \mathbb{R}, j=1,2$. Since $\mu_{2}^{2}-\mu_{1}^{2}=k_{2}^{2}-k_{1}^{2}$, we have

$$
p_{2}^{2}-q_{2}^{2}=k_{2}^{2}-k_{1}^{2}+p_{1}^{2}-p_{2}^{2}, \quad p_{1} q_{1}=p_{2} q_{2} .
$$


We recall (2.3) and find

$$
\begin{aligned}
& p_{2}=\left[\frac{\sqrt{\left(k_{2}^{2}-k_{1}^{2}+p_{1}^{2}-q_{1}^{2}\right)^{2}+4 p_{1}^{2} q_{1}^{2}}+\left(k_{2}^{2}-k_{1}^{2}+p_{1}^{2}-q_{1}^{2}\right)}{2}\right]^{1 / 2} \\
& q_{2}=\operatorname{sgn}\left(q_{1}\right)\left[\frac{\sqrt{\left(k_{2}^{2}-k_{1}^{2}+p_{1}^{2}-q_{1}^{2}\right)^{2}+4 p_{1}^{2} q_{1}^{2}}-\left(k_{2}^{2}-k_{1}^{2}+p_{1}^{2}-q_{1}^{2}\right)}{2}\right]^{1 / 2}
\end{aligned}
$$

Direct calculations show that

$$
p_{2} \geq p_{1} \geq 0, \quad\left|q_{2}\right| \leq\left|q_{1}\right|,
$$

where we have used $p_{1} \geq 0$ from Lemma 6.2. Since

$$
\operatorname{Im}\left[\left(\mu_{1}-\mu_{2}\right) z_{2}\right]=a_{2}\left(q_{1}-q_{2}\right)+b_{2}\left(p_{1}-p_{2}\right),
$$

the lemma now follows obviously for $q_{1} \leq 0$. The rest of the proof is devoted to the case of $q_{1}>0$.

By the assumption (6.10) we know that $\operatorname{Re}\left(z_{1}^{2}+z_{2}^{2}\right) \leq 0$. Thus (2.3) implies $\rho_{1} \leq \rho_{2}$, where $\rho_{1}=\operatorname{Re} \rho, \rho_{2}=\operatorname{Im} \rho$. By Lemma 6.2 we know that

$$
\begin{aligned}
& p_{1}=\frac{k_{1}}{|\rho|^{2}}\left[t\left(\rho_{1} a_{2}+\rho_{2} b_{2}\right)+t^{\prime}\left(\rho_{1} b_{1}-\rho_{2} a_{1}\right)\right], \\
& q_{1}=\frac{k_{1}}{|\rho|^{2}}\left[t\left(\rho_{1} b_{2}-\rho_{2} a_{2}\right)-t^{\prime}\left(\rho_{1} a_{1}+\rho_{2} b_{1}\right)\right] .
\end{aligned}
$$

This yields

$$
\begin{aligned}
p_{1}-q_{1} & =\frac{k_{1}}{|\rho|^{2}}\left\{t\left[a_{2}\left(\rho_{2}+\rho_{1}\right)+b_{2}\left(\rho_{2}-\rho_{1}\right)\right]+t^{\prime}\left[b_{1}\left(\rho_{2}+\rho_{1}\right)+a_{1}\left(\rho_{1}-\rho_{2}\right)\right]\right\} \\
& \geq \frac{k_{1} t^{\prime}\left(\rho_{2}-\rho_{1}\right)}{|\rho|^{2}}\left(a_{2}+b_{2}+b_{1}-a_{1}\right) \geq 0 .
\end{aligned}
$$

Since $q_{1}>0$, (6.16) implies that $\rho_{1} b_{2}>\rho_{2} a_{2}$, which together with $\rho_{1} \leq \rho_{2}$ implies $b_{2} \geq a_{2}$. Therefore, by (6.14), $q_{1}>0$, and $p_{1} q_{1}=p_{2} q_{2}$,

$$
\begin{aligned}
\operatorname{Im}\left[\left(\mu_{1}-\mu_{2}\right) z_{2}\right]=\frac{1}{q_{1}}\left(q_{2}-q_{1}\right)\left(b_{2} p_{2}-a_{2} q_{1}\right) & \leq \frac{a_{2}}{q_{1}}\left(q_{2}-q_{1}\right)\left(p_{2}-q_{1}\right) \\
& \leq \frac{a_{2}}{q_{1}}\left(q_{2}-q_{1}\right)\left(p_{1}-q_{1}\right) \leq 0,
\end{aligned}
$$

where we have used (6.13). This completes the proof. $\square$

Lemma 6.4. For $z_{1}, z_{2} \in \mathbb{C}$ and $\rho=\left(z_{1}^{2}+z_{2}^{2}\right)^{1 / 2}$. Suppose $k_{1} \operatorname{Im} \rho \geq 1$ and let $h(t)$ be a bounded function in $[1, \infty)$. Then the function

$$
\Phi_{1}\left(h ; z_{1}, z_{2}\right)=\frac{1}{2 \pi} \int_{1}^{\infty} \frac{h(t)}{\sqrt{t^{2}-1}} e^{\mathrm{i} k_{1} \rho t} \mathrm{~d} t
$$

satisfies the estimate

$$
\left|\Phi_{1}\left(h ; z_{1}, z_{2}\right)\right| \leq C M(h) e^{-k_{1} \operatorname{Im} \rho}
$$


where $M(h)$ is the upper bound of $|h|$ and $C$ is independent of $k_{1}, z_{1}, z_{2}$.

Proof. Since $k_{1} \operatorname{Im} \rho \geq 1$, we have

$$
\begin{aligned}
\left|\Phi_{1}\left(h ; z_{1}, z_{2}\right)\right| & \leq C M(h) \int_{1}^{\infty} \frac{1}{\sqrt{t^{2}-1}} e^{-k_{1} \operatorname{Im} \rho t} \mathrm{~d} t \\
& \leq C M(h) e^{-\left(k_{1} \operatorname{Im} \rho-1\right)} \int_{1}^{\infty} \frac{e^{-t}}{\sqrt{t^{2}-1}} \mathrm{~d} t \\
& =C M(h) e^{-k_{1} \operatorname{Im} \rho} .
\end{aligned}
$$

This completes the proof. $\square$

In the following we will always denote

$$
z_{1}=\left[\left(\tilde{x}_{1}-y_{1}\right)^{2}\right]^{1 / 2}, \quad z_{2}=\left[\left(\tilde{x}_{2}\right)^{2}\right]^{1 / 2}+\left|y_{2}\right|, \quad \forall x \in \Gamma_{2}, y \in \Gamma_{1} .
$$

Let $z_{j}=a_{j}+\mathbf{i} b_{j}, a_{j}, b_{j} \in \mathbb{R}, j=1,2$. Then $a_{j}, b_{j} \geq 0$. By Lemma 6.1. $\rho(\tilde{x}, y)=$ $\left(z_{1}^{2}+z_{2}^{2}\right)^{1 / 2}$ satisfies

$$
\begin{aligned}
\operatorname{Im} \rho(\tilde{x}, y) \geq & \frac{\left|x_{1}-y_{1}\right|}{\sqrt{\left|x_{1}-y_{1}\right|^{2}+\left(\left|x_{2}\right|+\left|y_{2}\right|\right)^{2}}}\left|\int_{0}^{x_{1}} \sigma_{1}(t) \mathrm{d} t\right| \\
& +\frac{\left|x_{2}\right|+\left|y_{2}\right|}{\sqrt{\left|x_{1}-y_{1}\right|^{2}+\left(\left|x_{2}\right|+\left|y_{2}\right|\right)^{2}}}\left|\int_{0}^{x_{2}} \sigma_{2}(t) \mathrm{d} t\right| .
\end{aligned}
$$

Now by (H2) we have, for any $x \in \Gamma_{2}$ and $y \in \Gamma_{1}$,

$$
\operatorname{Im} \rho(\tilde{x}, y) \geq \gamma_{0} \bar{\sigma}, \quad \gamma_{0}=\frac{\min \left(d_{1}, d_{2}+L_{2} / 2\right)}{\sqrt{\left(L_{1}+d_{1}\right)^{2}+\left(L_{2}+d_{2}\right)^{2}}} .
$$

The following lemma on the estimate of the modified Green function $\tilde{G}$ will play an important role in the following analysis.

Lemma 6.5. Let (H2) and $\gamma_{0} \bar{\sigma} \geq \max \left(k_{1}^{-1}, \min \left(d_{1}, d_{2}+L_{2} / 2\right)\right)$ be satisfied. There exists a constant $C$ independent of $k_{j}, L_{j}$, and $d_{j}, j=1,2$, such that

$$
|\tilde{G}(x, y)| \leq C \gamma_{1} e^{-k_{1} \gamma_{0} \bar{\sigma}}, \quad \forall x \in \Gamma_{2}, \quad y \in \Gamma_{1},
$$

where $\gamma_{1}:=e^{L_{2} \sqrt{k_{2}^{2}-k_{1}^{2}} / 2}$.

Before we prove the lemma we remark that the condition

$$
\gamma_{0} \bar{\sigma} \geq \max \left(k_{1}^{-1}, \min \left(d_{1}, d_{2}+L_{2} / 2\right)\right)
$$

in the lemma is rather mild in practical applications because one achieves the exponential convergence of the PML method by enlarging $\bar{\sigma}$ which can be realized by either enlarging the thickness of the PML layer $d_{j}$ or enlarging the medium property $\tilde{\sigma}_{j}$. Moreover, the condition $\gamma_{0} \bar{\sigma} \geq \min \left(d_{1}, d_{2}+L_{2} / 2\right)$ implies $b_{1}^{2}+b_{2}^{2} \geq a_{1}^{2}+a_{2}^{2}$ which is the condition (6.10) in Lemma 6.3.

Proof. We only prove the case for $y \in \mathbb{R}_{+}^{2}$, the proof of the other case $y \in \mathbb{R}_{-}^{2}$ is similar. By (2.5) and the method of Cagniard-de Hoop transform (cf. 9]) we know the Green function $\Phi\left(k_{1}, x, y\right)=\frac{\mathbf{i}}{4} H_{0}^{(1)}\left(k_{1}|x-y|\right)$ satisfies

$$
\Phi\left(k_{1}, x, y\right)=\frac{1}{2 \pi} \int_{1}^{\infty} \frac{1}{\sqrt{t^{2}-1}} e^{\mathrm{i} k_{1}|x-y| t} d t .
$$


Thus by (2.11) and Lemma 2.1. Lemma 6.4, we know that, for $x_{2}>0$,

$$
\tilde{G}(x, y)=\Phi_{1}\left(1 ; z_{1}, z_{2}^{\prime}\right)-\Phi_{1}\left(1 ; z_{1}, z_{2}\right)+\Phi_{1}\left(h_{1} ; z_{1}, z_{2}\right),
$$

where $z_{1}, z_{2}$ are given in (6.17), $z_{2}^{\prime}=\tilde{x}_{2}-y_{2}$, and

$$
h_{1}(t)=\operatorname{Re}\left(\frac{2 \mu_{1}}{\mu_{1}+\mu_{2}}\right) \text {. }
$$

Since $\operatorname{Im}\left(\mu_{1}^{2}\right)=\operatorname{Im}\left(\mu_{2}^{2}\right)$, by (2.3), $\operatorname{sgn}\left(\operatorname{Im} \mu_{1}\right)=\operatorname{sgn}\left(\operatorname{Im} \mu_{2}\right)$. Recalling the convention of choosing the analytic branch of $\sqrt{z}$, we know $\operatorname{Re} \mu_{1} \geq 0, \operatorname{Re} \mu_{2} \geq 0$. It follows that

$$
\left|\frac{\mu_{1}}{\mu_{1}+\mu_{2}}\right| \leq 1,
$$

which yields $\left|h_{1}(t)\right| \leq 2$. Then using (6.19), Lemma 6.4, and (6.18), we have

$$
|\tilde{G}(x, y)| \leq C e^{-k_{1} \gamma_{0} \bar{\sigma}}, \text { for } x_{2}>0 .
$$

Now we consider the case $x \in \mathbb{R}_{-}^{2}$. By (2.11), Lemma 2.1, and Lemma 6.4, we know that

$$
\tilde{G}(x, y)=\Phi_{1}\left(h_{2} ; z_{1}, z_{2}\right)
$$

where

$$
h_{2}:=\operatorname{Re}\left(\frac{2 \mu_{1}}{\mu_{1}+\mu_{2}} e^{\mathbf{i}\left(\mu_{1}-\mu_{2}\right)\left(\tilde{x}_{2}-y_{2}\right)} e^{\mathbf{i} y_{2}\left(\mu_{1}-\mu_{2}\right)}\right) .
$$

Let $\mu_{j}=p_{j}+\mathbf{i} q_{j}$ with $p_{j}, q_{j} \in \mathbb{R}, j=1,2$. By the remark after this lemma we can use Lemma 6.3 and (6.21) to obtain

$$
\left|h_{2}(t)\right| \leq 2 e^{\left|q_{1}-q_{2}\right|\left|y_{2}\right|}
$$

Since $q_{1}$ and $q_{2}$ have the same sign, we deduce that

$$
\left|q_{1}-q_{2}\right| \leq\left|\mu_{1}-\mu_{2}\right|=\frac{k_{2}^{2}-k_{1}^{2}}{\left|\mu_{1}+\mu_{2}\right|} \leq \frac{k_{2}^{2}-k_{1}^{2}}{\left|q_{1}+q_{2}\right|} \leq \frac{k_{2}^{2}-k_{1}^{2}}{\left|q_{1}-q_{2}\right|} .
$$

Then

$$
\left|h_{2}(t)\right| \leq e^{\sqrt{k_{2}^{2}-k_{1}^{2}}\left|y_{2}\right|} \leq e^{L_{2} \sqrt{k_{2}^{2}-k_{1}^{2}} / 2}, \quad \forall y \in \Gamma_{2} .
$$

This completes the proof by (6.22) and Lemma 6.4,

To estimate the derivatives of the modified Green function, we need to estimate the lower bound of $\mu_{2}(\xi)$ for all $\xi$ in (6.2) with $z_{1}, z_{2}$ in (6.17). We distinguish several cases.

$1^{\circ}$ ) If $b_{1}=0$, we have $\left|x_{1}\right| \leq L_{1} / 2$. Then (H2) and the fact $x \in \Gamma_{2}$ indicate that $b_{2}=\bar{\sigma}$. It follows from $\gamma_{0} \bar{\sigma} \geq \min \left(d_{1}, d_{2}+L_{2} / 2\right)$ that $\bar{\sigma} \geq \sqrt{\left(L_{1}+d_{1}\right)^{2}+\left(L_{2}+d_{2}\right)^{2}}$. Thus

$$
\left|z_{2}\right| \geq \bar{\sigma} \geq d_{1}+L_{1} \geq\left|x_{1}-y_{1}\right|=\left|z_{1}\right|, \quad \forall x \in \Gamma_{2}, y \in \Gamma_{1} .
$$


Since $|\beta| \leq 1 / 2$ by Cauchy-Schwarz inequality, we have $f_{2}(t) \geq 0$ for any $t \in[1, \infty)$ and consequently we deduce from (6.5) that

$$
\left|\mu_{2}\right| \geq \sqrt{\operatorname{Re}\left(k_{2}^{2}-\xi^{2}\right)} \geq \sqrt{k_{2}^{2}-\frac{1}{2} k_{1}^{2}} \geq \frac{1}{\sqrt{2}} k_{2} .
$$

$2^{\circ}$ ) If $b_{1}>0$ and $b_{2}>0$, then

$$
\text { either }\left|x_{1}\right|=L_{1} / 2+d_{1},\left|x_{2}\right| \geq L_{2} / 2 \text { or }\left|x_{2}\right|=L_{2} / 2+d_{2},\left|x_{1}\right| \geq L_{1} / 2 \text {. }
$$

Thus $a_{2}=\left|x_{2}\right|+\left|y_{2}\right| \geq L_{2} / 2$. If $\left|x_{1}\right|=L_{1} / 2+d_{1}, a_{1}=\left|x_{1}-y_{1}\right| \geq d_{1}$ for any $y \in \Gamma_{1}$. In the other case, $\left|x_{2}\right|=L_{2} / 2+d_{2}$ and hence $b_{2}=\bar{\sigma}$. If $\left|z_{2}\right| \leq\left|z_{1}\right|$, then $a_{2}^{2}+b_{2}^{2} \leq a_{1}^{2}+b_{1}^{2}$ and consequently $a_{1}^{2} \geq a_{2}^{2}+b_{2}^{2}-b_{1}^{2}=a_{2}^{2}+\bar{\sigma}^{2}-b_{1}^{2} \geq a_{2}^{2}$. That is $a_{1} \geq a_{2} \geq L_{2} / 2$. From (6.8) we have

$$
\left|\mu_{2}\right| \geq\left(k_{2}^{4}-k_{2}^{2} k_{1}^{2}\right)^{1 / 4} \frac{\sqrt{\min \left(d_{1}, L_{2} / 2\right) L_{2}}}{|\rho|} .
$$

On the other hand, if $\left|z_{2}\right| \geq\left|z_{1}\right|$, we obtain as in (6.23) that $\left|\mu_{2}\right| \geq \frac{1}{\sqrt{2}} k_{2}$. In summary we have

$$
\left|\mu_{2}\right| \geq \frac{1}{\sqrt{2}} k_{2} \quad \text { or } \quad\left|\mu_{2}\right| \geq\left(k_{2}^{4}-k_{2}^{2} k_{1}^{2}\right)^{1 / 4} \frac{\sqrt{\min \left(d_{1}, L_{2} / 2\right) L_{2}}}{|\rho|} .
$$

The trickiest case of $b_{2}=0$ is the objective of next lemma.

Lemma 6.6. Suppose $b_{2}=0$ and $b_{1}^{2}+b_{2}^{2} \geq a_{1}^{2}+a_{2}^{2}$. Define

$$
R=\sqrt{\frac{\left(t-t_{0}\right)^{2}}{2\left(t_{0}^{2}-1\right)^{2}}+\frac{a_{2}^{2}}{b_{1}^{2}}}
$$

where $t_{0}=k_{2} / k_{1}$. Then

$$
\left|\mu_{2}\right| \geq \sqrt{k_{2}^{2}-k_{1}^{2}} \frac{\sqrt{a_{1} b_{1}}}{|\rho|} \min (1, \sqrt{R}), \quad \forall t \in[1, \infty) .
$$

Proof. For $b_{2}=0$, we note that (6.8) becomes

$$
k_{1}^{-4}|\rho|^{4}\left|\mu_{2}(t)\right|^{4}=4\left(t_{0}^{4}-t_{0}^{2}\right) a_{1}^{2} a_{2}^{2}+\frac{1}{4}\left(\left|z_{1}\right|^{2}+\left|z_{2}\right|^{2}\right)^{2}\left(f_{2}(t)-M\right)^{2} .
$$

By (6.5) we have

$$
f_{2}(t)-M=2\left(t^{2}-t_{0}^{2}-2 \beta t t^{\prime}\right)+2\left(2 t_{0}^{2}-1\right) \frac{\left|z_{2}\right|^{2}}{\left|z_{1}\right|^{2}+\left|z_{2}\right|^{2}} .
$$

Note the elementary inequality

$$
(X+B)^{2} \geq(1-\varepsilon) X^{2}+\left(1-\varepsilon^{-1}\right) B^{2}, \quad \forall \varepsilon>0 .
$$

By taking $X=t^{2}-t_{0}^{2}-2 \beta t t^{\prime}, B=\frac{\left(2 t_{0}^{2}-1\right)\left|z_{2}\right|^{2}}{\left|z_{1}\right|^{2}+\left|z_{2}\right|^{2}}$, we know from (6.26) that

$$
\frac{1}{4}\left(f_{2}(t)-M\right)^{2} \geq(1-\varepsilon)\left(t^{2}-t_{0}^{2}-2 \beta t t^{\prime}\right)^{2}+\left(1-\varepsilon^{-1}\right)\left(2 t_{0}^{2}-1\right)^{2} \frac{\left|z_{2}\right|^{4}}{\left(\left|z_{1}\right|^{2}+\left|z_{2}\right|^{2}\right)^{2}}
$$


Again using (6.26) with $\varepsilon=1 / 2$, we have, for $1 \leq t \leq t_{0}$, that

$$
\left(t^{2}-t_{0}^{2}-2 \beta t t^{\prime}\right)^{2} \geq \frac{1}{2}\left(t^{2}-t_{0}^{2}\right)^{2}-4 \beta^{2} t^{2}{t^{\prime}}^{2} \geq\left(t-t_{0}\right)^{2}-\frac{4 a_{2}^{2} b_{1}^{2}}{\left(\left|z_{1}\right|^{2}+\left|z_{2}\right|^{2}\right)^{2}}\left(t_{0}^{4}-t_{0}^{2}\right) .
$$

Now since $b_{2}=0, \beta \leq 0$ by (6.6). The above inequality is also valid for $t \geq t_{0} \geq 1$ because $t^{2}-t_{0}^{2}-2 \beta t t^{\prime} \geq t^{2}-t_{0}^{2} \geq t-t_{0} \geq 0$ for $t \geq t_{0}$.

Using (6.25) we have

$$
\begin{aligned}
k_{1}^{-4}|\rho|^{4}\left|\mu_{2}\right|^{4} \geq & (1-\varepsilon)\left(\left|z_{1}\right|^{2}+\left|z_{2}\right|^{2}\right)^{2}\left(t-t_{0}\right)^{2}+\left(1-\varepsilon^{-1}\right)\left(2 t_{0}^{2}-1\right)^{2} a_{2}^{4} \\
& +4 a_{2}^{2}\left(t_{0}^{4}-t_{0}^{2}\right)\left[a_{1}^{2}-(1-\varepsilon) b_{1}^{2}\right] .
\end{aligned}
$$

By the assumption $b_{1}^{2}+b_{2}^{2} \geq a_{1}^{2}+a_{2}^{2}$ and $b_{2}=0$, we have $b_{1}^{2} \geq a_{1}^{2}$. Therefore we can set $\varepsilon=1-a_{1}^{2} /\left(2 b_{1}^{2}\right)>0$ in above inequality to obtain

$$
k_{1}^{-4}|\rho|^{4}\left|\mu_{2}\right|^{4} \geq \frac{a_{1}^{2}}{2 b_{1}^{2}}\left(\left|z_{1}\right|^{2}+\left|z_{2}\right|^{2}\right)^{2}\left(t-t_{0}\right)^{2}+a_{1}^{2} a_{2}^{2}\left[2\left(t_{0}^{4}-t_{0}^{2}\right)-\frac{a_{2}^{2}}{b_{1}^{2}}\left(2 t_{0}^{2}-1\right)^{2}\right],
$$

where we have used the fact that $1-\varepsilon^{-1}=-a_{1}^{2} /\left(2 b_{1}^{2}-a_{1}^{2}\right) \geq-a_{1}^{2} / b_{1}^{2}$.

If $t_{0}^{4}-t_{0}^{2} \geq \frac{a_{2}^{2}}{b_{1}^{2}}\left(2 t_{0}^{2}-1\right)^{2}$, since $\left|z_{1}\right|^{2}+\left|z_{2}\right|^{2} \geq b_{1}^{2}$, we have

$$
k_{1}^{-4}|\rho|^{4}\left|\mu_{2}\right|^{4} \geq \frac{1}{2} a_{1}^{2} b_{1}^{2}\left(t-t_{0}\right)^{2}+a_{1}^{2} a_{2}^{2}\left(t_{0}^{4}-t_{0}^{2}\right)=a_{1}^{2} b_{1}^{2}\left(t_{0}^{2}-1\right)^{2} R^{2} .
$$

If $t_{0}^{4}-t_{0}^{2} \leq \frac{a_{2}^{2}}{b_{1}^{2}}\left(2 t_{0}^{2}-1\right)^{2}$ which is equivalent to $a_{2}^{2}>\frac{t_{0}^{4}-t_{0}^{2}}{\left(2 t_{0}^{2}-1\right)^{2}} b_{1}^{2}$, we deduce from (6.25) that

$$
k_{1}^{-4}|\rho|^{4}\left|\mu_{2}(t)\right|^{4} \geq 4\left(t_{0}^{4}-t_{0}^{2}\right) a_{1}^{2} \frac{\left(t_{0}^{4}-t_{0}^{2}\right)^{2}}{\left(2 t_{0}^{2}-1\right)^{2}} b_{1}^{2} \geq\left(t_{0}^{2}-1\right)^{2} a_{1}^{2} b_{1}^{2} .
$$

This completes the proof.

Lemma 6.7. Let (H2) and $\gamma_{0} \bar{\sigma} \geq \max \left(k_{1}^{-1}, \min \left(d_{1}, d_{2}+L_{2} / 2\right)\right)$ be satisfied. There exists a constant $C$ depending only on $\gamma_{0}, k_{2} / k_{1}, L_{2} / L_{1}$ but independent of $k_{j}, L_{j}$, and $d_{j}, j=1,2$, such that, for any $x \in \Gamma_{2}$ and $y \in \Gamma_{1}$,

$$
\begin{aligned}
& \left|\frac{\partial \tilde{G}(x, y)}{\partial y_{j}}\right| \leq C \gamma_{1} k_{1}\left(1+\frac{1}{k_{1} L_{1}}\right) e^{-k_{1} \gamma_{0} \bar{\sigma}}, \\
& \left|\frac{\partial \tilde{G}(x, y)}{\partial x_{j}}\right| \leq C \gamma_{1} k_{1} \alpha_{m}\left(1+\frac{1}{k_{1} L_{1}}\right) e^{-k_{1} \gamma_{0} \bar{\sigma}} .
\end{aligned}
$$

Here $\alpha_{m}=\max _{x \in \Omega_{\mathrm{PML}}}\left(\left|\alpha_{1}\left(x_{1}\right)\right|,\left|\alpha_{2}\left(x_{2}\right)\right|\right), \gamma_{1}$ is defined in Lemma 6.5.

Proof. By the symmetry of the Green function $G(x, y)$ we know that $\frac{\partial G(\tilde{x}, y)}{\partial x_{j}}=$ $-\alpha\left(x_{j}\right) \frac{\partial G(\tilde{x}, y)}{\partial y_{j}}$. Thus we only need to prove (6.28) which will be given only for $x, y \in \mathbb{R}_{+}^{2}$. The proof for other cases is similar.

In view of (6.19), we have, for any $x, y \in \mathbb{R}_{+}^{2}$,

$$
\left|\frac{\partial \tilde{G}(x, y)}{\partial y_{j}}\right| \leq\left|\frac{\partial \Phi_{1}}{\partial z_{j}}\left(1 ; z_{1}, z_{2}^{\prime}\right)\right|+\left|\frac{\partial \Phi_{1}}{\partial z_{j}}\left(1 ; z_{1}, z_{2}\right)\right|+\left|\frac{\partial \Phi_{1}}{\partial z_{j}}\left(h_{1} ; z_{1}, z_{2}\right)\right| .
$$


Since the estimation for $\frac{\partial \Phi_{1}}{\partial z_{j}}\left(1 ; z_{1}, z_{2}^{\prime}\right)$ and $\frac{\partial \Phi_{1}}{\partial z_{j}}\left(1 ; z_{1}, z_{2}\right)$ is simpler, we consider only $\frac{\partial \Phi_{1}}{\partial z_{j}}\left(h_{1} ; z_{1}, z_{2}\right)$ in the sequel. that

From the definition of $\Phi_{1}\left(h_{1} ; z_{1}, z_{2}\right)$ in Lemma 6.4 and $\partial \rho / \partial z_{j}=z_{j} / \rho$ we know

$$
\frac{\partial \Phi_{1}}{\partial z_{j}}\left(h_{1} ; z_{1}, z_{2}\right)=\Phi_{1}\left(\frac{\partial h_{1}}{\partial z_{j}}+\mathbf{i} k_{1} h_{1} \frac{z_{j}}{\rho} t ; z_{1}, z_{2}\right), j=1,2 .
$$

Since the remark below Lemma 6.5 we know that the assumption of the lemma implies $b_{1}^{2}+b_{2}^{2} \geq a_{1}^{2}+a_{2}^{2}$, by (6.18) and the fact that $\left|b_{j}\right| \leq \bar{\sigma}$ for $x \in \Gamma_{2}, y \in \Gamma_{1}$, we know that

$$
\left|\frac{z_{j}}{\rho}\right| \leq \frac{\sqrt{a_{j}^{2}+b_{j}^{2}}}{\gamma_{0} \bar{\sigma}} \leq \frac{\sqrt{3}}{\gamma_{0}} .
$$

Recall Lemma 6.2 for the expressions of $\xi$ and $\mu_{1}$. There exists a constant depending only on $\gamma_{0}$ but independent of $k_{j}, d_{j}, L_{j}, j=1,2$, such that

$$
\left|\frac{\partial \xi}{\partial z_{j}}\right|+\left|\frac{\partial \mu_{1}}{\partial z_{j}}\right| \leq C \frac{k_{1} t}{|\rho|^{j}}, \quad j=0,1,2 .
$$

By the chain rule $\frac{\partial \mu_{2}}{\partial z_{j}}=\frac{\mu_{1}}{\mu_{2}} \frac{\partial \mu_{1}}{\partial z_{j}}$, we deduce by direct calculation and using (6.32) that

$$
\left|\frac{\partial h_{1}}{\partial z_{j}}\right| \leq C\left|\frac{\mu_{1}-\mu_{2}}{\mu_{2}\left(\mu_{1}+\mu_{2}\right)}\right|\left|\frac{\partial \mu_{1}}{\partial z_{j}}\right| \leq C \frac{k_{1} t}{|\rho|\left|\mu_{2}\right|}
$$

where we have used $\left|\frac{\mu_{1}-\mu_{2}}{\mu_{1}+\mu_{2}}\right| \leq 1$. Then by using (6.30)-(6.31) and Lemma 6.4 we have that

$$
\left|\frac{\partial \Phi_{1}}{\partial z_{j}}\left(h_{1} ; z_{1}, z_{2}\right)\right| \leq C k_{1} e^{-k_{1} \gamma_{0} \bar{\sigma}}\left(1+K_{1}\right), \quad K_{1}=\int_{1}^{\infty} \frac{t e^{-t}}{|\rho|\left|\mu_{2}\right| \sqrt{t^{2}-1}} \mathrm{~d} t .
$$

To estimate $K_{1}$, notice that under the assumption $\gamma_{0} \bar{\sigma} \geq \min \left(d_{1}, d_{2}+L_{2} / 2\right), \bar{\sigma} \geq$ $\sqrt{\left(L_{1}+d_{1}\right)^{2}+\left(L_{2}+d_{2}\right)^{2}}$. Thus $|\rho| \geq \gamma_{0} \bar{\sigma} \geq C L_{1}$. Now by (6.23)-(6.24) and Lemma 6.6 we have

$$
|\rho|\left|\mu_{2}\right| \geq C k_{1} L_{1} \min \left(1, \sqrt{\left|t-t_{0}\right|}\right),
$$

which implies easily $\left|K_{1}\right| \leq C /\left(k_{1} L_{1}\right)$. Thus

$$
\left|\frac{\partial \Phi_{1}}{\partial z_{j}}\left(h_{1} ; z_{1}, z_{2}\right)\right| \leq C k_{1}\left(1+\frac{1}{k_{1} L_{1}}\right) e^{-k_{1} \gamma_{0} \bar{\sigma}} .
$$

This completes the proof. $\square$

Lemma 6.8. Let (H2) and $\gamma_{0} \bar{\sigma} \geq \max \left(k_{1}^{-1}, \min \left(d_{1}, d_{2}+L_{2} / 2\right)\right)$ be satisfied. There exists a constant $C$ depending only on $\gamma_{0}, k_{2} / k_{1}, L_{2} / L_{1}$ but independent of $k_{j}, L_{j}$, and $d_{j}, j=1,2$, such that, for any $x \in \Gamma_{2}$ and $1 \leq p<4 / 3$,

$$
\left\|\frac{\partial^{2} \tilde{G}(x, \cdot)}{\partial x_{i} \partial y_{j}}\right\|_{L^{p}\left(\Gamma_{1}\right)} \leq C \gamma_{1} k_{1}^{2} L_{1}^{1 / p}\left(1+\frac{1}{k_{1} L_{1}}\right)^{2}\left(1+\frac{\bar{\sigma}}{L_{1}}\right)^{2} \alpha_{m} e^{-k_{1} \gamma_{0} \bar{\sigma}} .
$$


Proof. We start by estimating $\frac{\partial^{2} \tilde{G}}{\partial x_{i} \partial y_{j}}(x, y)$ for any $x \in \Gamma_{2}$ and $y \in \Gamma_{1}$. We first consider the case when $x, y \in \mathbb{R}_{+}^{2}$. In view of (6.19), we have, for any $x, y \in \mathbb{R}_{+}^{2}$,

$$
\left|\frac{\partial^{2} \tilde{G}(x, y)}{\partial x_{i} \partial y_{j}}\right| \leq\left|\alpha_{i}\right|\left(\left|\frac{\partial^{2} \Phi_{1}}{\partial z_{i} \partial z_{j}}\left(1 ; z_{1}, z_{2}^{\prime}\right)\right|+\left|\frac{\partial^{2} \Phi_{1}}{\partial z_{i} \partial z_{j}}\left(1 ; z_{1}, z_{2}\right)\right|+\left|\frac{\partial^{2} \Phi_{1}}{\partial z_{i} \partial z_{j}}\left(h_{1} ; z_{1}, z_{2}\right)\right|\right) \text {. }
$$

Since the estimation for $\frac{\partial^{2} \Phi_{1}}{\partial z_{i} \partial z_{j}}\left(1 ; z_{1}, z_{2}^{\prime}\right)$ and $\frac{\partial^{2} \Phi_{1}}{\partial z_{i} \partial z_{j}}\left(1 ; z_{1}, z_{2}\right)$ is simpler, we only consider $\frac{\partial^{2} \Phi_{1}}{\partial z_{i} \partial z_{j}}\left(h_{1} ; z_{1}, z_{2}\right)$ in the sequel.

It is easy to see that

$$
\begin{aligned}
\frac{\partial^{2} \Phi_{1}}{\partial z_{i} \partial z_{j}}\left(h_{1} ; z_{1}, z_{2}\right)= & \Phi_{1}\left(\frac{\partial^{2} h_{1}}{\partial z_{i} \partial z_{j}}+\mathbf{i} k_{1} t \frac{\partial}{\partial z_{i}}\left(h_{1} \frac{z_{j}}{\rho}\right) ; z_{1}, z_{2}\right) \\
& +\Phi_{1}\left(\left(\frac{\partial h_{1}}{\partial z_{j}}+\mathbf{i} k_{1} h_{1} \frac{z_{j}}{\rho} t\right) \mathbf{i} k_{1} t \frac{z_{i}}{\rho} ; z_{1}, z_{2}\right) .
\end{aligned}
$$

By the chain rule $\frac{\partial \mu_{2}}{\partial z_{j}}=\frac{\mu_{1}}{\mu_{2}} \frac{\partial \mu_{1}}{\partial z_{j}}$ and (6.32), there exists a constant $C$ depending only on $\gamma_{0}, k_{2} / k_{1}$ but independent of $k_{j}$ and $d_{j}, j=1,2$, such that

$$
\begin{aligned}
\left|\frac{\partial^{2} h_{1}}{\partial z_{i} \partial z_{j}}\right| & \leq\left|\frac{\mu_{1}-\mu_{2}}{\mu_{2}\left(\mu_{1}+\mu_{2}\right)}\right|\left|\frac{\partial^{2} \mu_{1}}{\partial z_{i} \partial z_{j}}\right|+\left|\frac{2 \mu_{2}+\mu_{1}}{\mu_{2}^{3}}\right|\left|\frac{\mu_{1}-\mu_{2}}{\mu_{1}+\mu_{2}}\right|\left|\frac{\partial \mu_{1}}{\partial z_{i}}\right|\left|\frac{\partial \mu_{1}}{\partial z_{j}}\right| \\
& \leq C \frac{k_{1} t}{|\rho|^{2}\left|\mu_{2}\right|}+C \frac{k_{1}^{3} t^{3}}{|\rho|^{2}\left|\mu_{2}\right|^{3}}
\end{aligned}
$$

where we have used $\left|\frac{\mu_{1}-\mu_{2}}{\mu_{1}+\mu_{2}}\right| \leq 1$ and $\left|\mu_{2}\right| \leq k_{2}+\left|\mu_{1}\right| \leq C k_{1} t$ for $t \geq 1$. By the assumption $\gamma_{0} \bar{\sigma} \geq \min \left(d_{1}, d_{2}+L_{2} / 2\right), k_{1}|\rho| \geq k_{1} \operatorname{Im} \rho \geq k_{1} \gamma_{0} \bar{\sigma} \geq 1$, we then have

$$
\left|\frac{\partial^{2} h_{1}}{\partial z_{i} \partial z_{j}}\right| \leq C \frac{k_{1}^{2} t}{|\rho|\left|\mu_{2}\right|}+C \frac{k_{1}^{3} t^{3}|\rho|}{|\rho|^{3}\left|\mu_{2}\right|^{3}} .
$$

Similarly, by using (6.33) we can obtain

$$
\begin{aligned}
& \left|\mathbf{i} k_{1} t \frac{\partial}{\partial z_{i}}\left(h_{1} \frac{z_{j}}{\rho}\right)\right| \leq C k_{1}^{2} t^{2} \frac{1}{|\rho|\left|\mu_{2}\right|}, \\
& \left|\left(\frac{\partial h_{1}}{\partial z_{j}}+\mathbf{i} k_{1} h_{1} \frac{z_{j}}{\rho} t\right) \mathbf{i} k_{1} t \frac{z_{i}}{\rho}\right| \leq C k_{1}^{2} t^{2}\left(1+\frac{1}{|\rho|\left|\mu_{2}\right|}\right) .
\end{aligned}
$$

By Lemma 6.4

$$
\left|\frac{\partial^{2} \Phi_{1}}{\partial z_{i} \partial z_{j}}\left(h_{1} ; z_{1}, z_{2}\right)\right| \leq C k_{1}^{2} e^{-k_{1} \gamma_{0} \bar{\sigma}}\left(1+K_{1}+K_{2}+K_{3}\right),
$$

where $K_{1}$ is defined in the proof of last lemma, and

$$
K_{2}:=\int_{1}^{\infty} \frac{t^{2} e^{-t}}{|\rho|\left|\mu_{2}\right| \sqrt{t^{2}-1}} \mathrm{~d} t, \quad K_{3}:=\int_{1}^{\infty} \frac{k_{1} t^{3}|\rho| e^{-t}}{|\rho|^{3}\left|\mu_{2}\right|^{3} \sqrt{t^{2}-1}} \mathrm{~d} t .
$$


The estimate (6.35) is also valid for the other cases when $x$ and $y$ are not both in $\mathbb{R}_{+}^{2}$ if the constant $C$ is replaced by $C \gamma_{1}$.

From the proof of last lemma we know that $\left|K_{1}\right| \leq C /\left(k_{1} L_{1}\right)$. Similarly we can prove $\left|K_{2}\right| \leq C /\left(k_{1} L_{1}\right)$. It remains to estimate $K_{3}$. For $\left|x_{2}\right|>L_{2} / 2$ we have $b_{2}>0$ and by (6.23) -(6.24) we know that $|\rho|\left|\mu_{2}\right| \geq C k_{1} L_{1}$ and thus $K_{3} \leq C k_{1}|\rho| /\left(k_{1} L_{1}\right)^{3}$ which yields

$$
\left.\left.\left|\int_{\Gamma_{1}}\right| K_{3}\right|^{p} d s(y)\right|^{1 / p} \leq C \frac{1}{k_{1}^{2} L_{1}^{2}}\left(\frac{\bar{\sigma}}{L_{1}}\right) L_{1}^{1 / p}
$$

For $\left|x_{2}\right| \leq L_{2} / 2$ we know that $b_{2}=0, b_{1}=\bar{\sigma}$, and $a_{1}=\left|x_{1}-y_{1}\right| \geq d_{1} \geq \gamma_{0}^{-1} L_{1}$, $a_{2}=\left|x_{2}\right|^{2}+y_{2}^{2} \geq y_{2}^{2}$. Thus by Lemma 6.6.

$$
|\rho|\left|\mu_{2}\right| \geq C k_{1} \sqrt{L_{1} \bar{\sigma}} \min (1, \sqrt{r}), \quad r=\sqrt{\left(t-t_{0}\right)^{2}+y_{2}^{2} / \bar{\sigma}^{2}} .
$$

Thus, since $|\rho| \leq C \bar{\sigma}, \bar{\sigma} \geq \sqrt{\left(L_{1}+d_{1}\right)^{2}+\left(L_{2}+d_{2}\right)^{2}}$,

$$
\left.\left.\left|\int_{\Gamma_{1}}\right| K_{3}\right|^{p} d s(y)\right|^{1 / p} \leq C \frac{1}{k_{1}^{2} L_{1}^{2}}\left(\int_{\Gamma_{1}}\left|\int_{1}^{\infty} \frac{t^{3} e^{-t}}{\min \left(1, r^{3 / 2}\right) \sqrt{t^{2}-1}} \mathrm{~d} t\right|^{p} \mathrm{~d} s(y)\right)^{1 / p} .
$$

Notice that

$$
r^{-3 / 2} \leq\left(\frac{2}{t_{0}-1}\right)^{3 / 2} \leq C, \quad \forall t \in\left[1, \frac{t_{0}+1}{2}\right] \cup\left[\frac{3 t_{0}-1}{2}, \infty\right) .
$$

An application of Holder's inequality yields that

$$
\begin{aligned}
\int_{\Gamma_{1}}\left|\int_{1}^{\infty} \frac{t^{3} e^{-t}}{r^{3 / 2} \sqrt{t^{2}-1}} \mathrm{~d} t\right|^{p} \mathrm{~d} s(y) & \leq C L_{1}+C \int_{\Gamma_{1}}\left|\int_{\frac{t_{0}+1}{2}}^{\frac{3 t_{0}-1}{2}} \frac{t^{3} e^{-t}}{r^{3 / 2} \sqrt{t^{2}-1}} \mathrm{~d} t\right|^{p} \mathrm{~d} s(y) \\
& \leq C L_{1}+C \int_{\Gamma_{1}} \int_{\frac{t_{0}+1}{2}}^{\frac{3 t_{0}-1}{2}} r^{-\frac{3 p}{2}} \mathrm{~d} t \mathrm{~d} s(y) .
\end{aligned}
$$

On the part of the boundary of $\Gamma_{1}$ where $\left|y_{2}\right|=L_{2} / 2$, we have $r^{-1} \leq \bar{\sigma} / L_{2}$, thus

$$
\begin{aligned}
\int_{\Gamma_{1}}\left|\int_{1}^{\infty} \frac{t^{3} e^{-t}}{r^{3 / 2} \sqrt{t^{2}-1}} \mathrm{~d} t\right|^{p} \mathrm{~d} s(y) & \leq C L_{1}+C L_{1}\left(\frac{\bar{\sigma}}{L_{2}}\right)^{\frac{3 p}{2}}+C \int_{0}^{\frac{L_{2}}{2}} \int_{\frac{t_{0}+1}{2}}^{\frac{3 t_{0}-1}{2}} r^{-\frac{3 p}{2}} \mathrm{~d} t \mathrm{~d} y_{2} \\
& \leq C L_{1}+C L_{1}\left(\frac{\bar{\sigma}}{L_{2}}\right)^{2}+C \bar{\sigma}, \quad \text { if } p<4 / 3 .
\end{aligned}
$$

This completes the proof. $\square$

7. The convergence. In this section, we are going to show the exponential convergence of the solution of (4.6) -4.7) to the solution of the original scattering problem (1.1)-(1.3). We first introduce the approximate Dirichlet-to-Neumann operator $\hat{T}: H^{1 / 2}\left(\Gamma_{1}\right) \rightarrow H^{-1 / 2}\left(\Gamma_{1}\right)$ associated with the UPML problem. Given $\zeta \in H^{1 / 2}\left(\Gamma_{1}\right)$, let $\hat{T} \zeta=\left.\frac{\partial \phi}{\partial \mathbf{n}}\right|_{\Gamma_{1}}$, where $\phi$ is the solution of the following Dirichlet problem in the PML layer:

$$
\begin{array}{ll}
\nabla \cdot(A \nabla \phi)+\alpha_{1} \alpha_{2} k^{2} \phi=0 & \text { in } \Omega_{\mathrm{PML}} \\
\phi=\zeta \quad \text { on } \Gamma_{1}, \quad \phi=0 \quad \text { on } \Gamma_{2} .
\end{array}
$$


From (5.4) we know that (7.1) -(7.2) has a unique solution and thus $\hat{T}$ is well-defined. Then (4.6) -(4.7) is reduced to the following weak formulation: Find $\hat{u} \in H^{1}\left(\Omega_{1}\right)$ such that $\hat{u}=g$ on $\Gamma_{D}$ and

$$
\hat{a}(\hat{u}, v)=(f, v), \quad \forall v \in H_{\Gamma_{D}}^{1}\left(\Omega_{1}\right),
$$

where the sesquilinear form $\hat{a}: H^{1}\left(\Omega_{1}\right) \times H^{1}\left(\Omega_{1}\right) \rightarrow \mathbb{C}$ is defined by

$$
\hat{a}(\varphi, \psi)=\int_{\Omega_{1}}\left(\nabla \varphi \cdot \nabla \bar{\psi}-k^{2} \varphi \bar{\psi}\right) \mathrm{d} x-\langle\hat{T} \varphi, \psi\rangle_{\Gamma_{1}}, \quad \forall \varphi, \psi \in H^{1}\left(\Omega_{1}\right) .
$$

We are in the position to estimate the error $T \zeta-\hat{T} \zeta$ for any $\zeta \in H^{1 / 2}\left(\Gamma_{1}\right)$. It is obvious that

$$
T \zeta-\hat{T} \zeta=\left.\frac{\partial w}{\partial \mathbf{n}}\right|_{\Gamma_{1}}
$$

where $w \in H^{1}\left(\Omega_{\mathrm{PML}}\right)$ is the solution of the Dirichlet problem (5.1)-(5.2) with $q=$ $\mathbb{E}(\zeta)$.

For any $\zeta \in H^{1 / 2}\left(\Gamma_{1}\right)$, denote

$$
X(\zeta):=\left\{v \in H^{1}\left(\Omega_{\mathrm{PML}}\right): v=0 \text { on } \Gamma_{1}, v=\mathbb{E}(\zeta) \text { on } \Gamma_{2}\right\} .
$$

By Lemma 5.2 we know that

$$
\|T \zeta-\hat{T} \zeta\|_{H^{-1 / 2}\left(\Gamma_{1}\right)} \leq\left(1+\hat{C}^{-1}\right) \inf _{v \in X(\zeta)}\|v\|_{*, \Omega_{\mathrm{PML}}}
$$

We introduce the weighted $H^{1 / 2}\left(\Gamma_{j}\right)$-norm, $j=1,2$,

$$
\|v\|_{H^{1 / 2}\left(\Gamma_{j}\right)}:=\left(\left|\Gamma_{j}\right|^{-1}\|v\|_{L^{2}\left(\Gamma_{j}\right)}^{2}+|v|_{\frac{1}{2}, \Gamma_{j}}^{2}\right)^{1 / 2}, \quad \forall v \in H^{1 / 2}\left(\Gamma_{j}\right),
$$

where

$$
|v|_{\frac{1}{2}, \Gamma_{j}}^{2}:=\int_{\Gamma_{j}} \int_{\Gamma_{j}} \frac{\left|v(x)-v\left(x^{\prime}\right)\right|^{2}}{\left|x-x^{\prime}\right|^{2}} \mathrm{~d} s(x) \mathrm{d} s\left(x^{\prime}\right) .
$$

Lemma 7.1. Let (H2) and $\gamma_{0} \bar{\sigma} \geq \max \left(k_{1}^{-1}, \min \left(d_{1}, d_{2}+L_{2} / 2\right)\right)$ be satisfied. There exists a constant $C$ depending only on $\gamma_{0}, k_{2} / k_{1}, L_{2} / L_{1}$ but independent of $k_{j}, L_{j}$, and $d_{j}, j=1,2$, such that, for any $\zeta \in H^{1 / 2}\left(\Gamma_{1}\right)$,

$$
\inf _{v \in X(\zeta)}\|v\|_{*, \Omega_{\mathrm{PML}}} \leq C \gamma_{1}\left(1+k_{1} L_{1}\right)^{3} \alpha_{m}^{3}\left(1+\frac{\bar{\sigma}}{L_{1}}\right)^{2}\|\zeta\|_{H^{1 / 2}\left(\Gamma_{1}\right)} .
$$

Proof. By definition

$$
\begin{aligned}
\|v\|_{*, \Omega_{\mathrm{PML}}}^{2} & =\|A \nabla v\|_{L^{2}\left(\Omega_{\mathrm{PML}}\right)}^{2}+\left\|k \alpha_{1} \alpha_{2} v\right\|_{L^{2}\left(\Omega_{\mathrm{PML}}\right)}^{2} \\
& \leq C\left(1+k_{1} L_{1}\right)^{2} \alpha_{m}^{4}\left(\|\nabla v\|_{L^{2}\left(\Omega_{\mathrm{PML}}\right)}^{2}+\left|\Gamma_{1}\right|^{-2}\|v\|_{L^{2}\left(\Omega_{\mathrm{PML}}\right)}^{2}\right) .
\end{aligned}
$$

which by the trace inequality implies that

$$
\inf _{v \in X(\zeta)}\|v\|_{*, \Omega_{\mathrm{PML}}} \leq C\left(1+k_{1} L_{1}\right) \alpha_{m}^{2}\|\mathbb{E}(\zeta)\|_{H^{1 / 2}\left(\Gamma_{2}\right)} .
$$


The definition of the $H^{1 / 2}$-norm shows that

$$
\inf _{v \in X(\zeta)}\|v\|_{*, \Omega_{\mathrm{PML}}} \leq C\left(1+k L_{1}\right) \alpha_{m}^{2}\left(\|\mathbb{E}(\zeta)\|_{L^{\infty}\left(\Gamma_{2}\right)}+\left|\Gamma_{1}\right||\mathbb{E}(\zeta)|_{W^{1, \infty}\left(\Gamma_{2}\right)}\right) .
$$

On the other hand,

$$
\begin{aligned}
|\mathbb{E}(\zeta)| & =\left|-\tilde{\Psi}_{\mathrm{SL}}(\lambda)+\tilde{\Psi}_{\mathrm{DL}}(\zeta)\right| \\
& =\left|-\int_{\Gamma_{1}} \tilde{G}(x, y) \lambda(y) \mathrm{d} s(y)+\int_{\Gamma_{1}} \frac{\partial \tilde{G}(x, y)}{\partial \mathbf{n}_{1}(y)} \zeta(y) \mathrm{d} s(y)\right| \\
& \leq C\|\tilde{G}(x, \cdot)\|_{H^{1 / 2}\left(\Gamma_{1}\right)}\|\lambda\|_{H^{-1 / 2}\left(\Gamma_{1}\right)}+\left\|\partial \tilde{G}(x, \cdot) / \partial \mathbf{n}_{1}(y)\right\|_{L^{\infty}\left(\Gamma_{1}\right)}\|\zeta\|_{L^{1}\left(\Gamma_{1}\right)} .
\end{aligned}
$$

Since $\|\lambda\|_{H^{-1 / 2}\left(\Gamma_{1}\right)} \leq C\|\zeta\|_{H^{1 / 2}\left(\Gamma_{1}\right)}$, we then obtain

$$
|\mathbb{E}(\zeta)| \leq C\left(1+k_{1} L_{1}\right) \max _{y \in \Gamma_{1}}\left(|\tilde{G}(x, \cdot)|+L_{1}\left|\nabla_{y} \tilde{G}(x, \cdot)\right|\right)\|\zeta\|_{H^{1 / 2}\left(\Gamma_{1}\right)},
$$

which implies by Lemmas 6.5 and Lemma 6.7 that

$$
|\mathbb{E}(\zeta)| \leq C \gamma_{1}\left(1+k_{1} L_{1}\right)^{2} e^{-k_{1} \gamma_{0} \bar{\sigma}} .
$$

Similarly, for any $1<p<4 / 3$, we know from the embedding theorem that $W^{1, p}\left(\Gamma_{1}\right)$ is embedded to $H^{1 / 2}\left(\Gamma_{1}\right)$ and $H^{1 / 2}\left(\Gamma_{1}\right)$ is embedded to $L^{p^{\prime}}\left(\Gamma_{1}\right)$, where $1 / p+1 / p^{\prime}=1$. Then

$$
\begin{aligned}
\left|\nabla_{x} \mathbb{E}(\zeta)\right| & \leq\left\|\nabla_{x} \tilde{G}(x, \cdot)\right\|_{H^{1 / 2}\left(\Gamma_{1}\right)}\|\lambda\|_{H^{-1 / 2}\left(\Gamma_{1}\right)}+\left\|\nabla_{x} \nabla_{y} \tilde{G}(x, \cdot)\right\|_{L^{p}\left(\Gamma_{1}\right)}\|\zeta\|_{L^{1 / p^{\prime}}\left(\Gamma_{1}\right)} \\
& \leq C L_{1}^{-1 / p}\left(\left\|\nabla_{x} \tilde{G}(x, \cdot)\right\|_{L^{p}\left(\Gamma_{1}\right)}+L_{1}\left\|\nabla_{x} \nabla_{y} \tilde{G}(x, \cdot)\right\|_{L^{p}\left(\Gamma_{1}\right)}\right)\|\zeta\|_{H^{1 / 2}\left(\Gamma_{1}\right)} .
\end{aligned}
$$

This implies by using Lemmas $6.7,6.8$ that

$$
L_{1}\left|\nabla_{x} \mathbb{E}(\zeta)\right| \leq C \alpha_{m}\left(1+k_{1} L_{1}\right)^{2}\left(1+\frac{\bar{\sigma}}{L_{1}}\right)^{2} e^{-k_{1} \gamma_{0} \bar{\sigma}}\|\zeta\|_{H^{1 / 2}\left(\Gamma_{1}\right)} .
$$

This competes the proof.

Now we are ready to present the main result of this paper.

TheOREM 7.2. Let (H1)-(H2) and $\gamma_{0} \bar{\sigma} \geq \max \left(k_{1}^{-1}, \min \left(d_{1}, d_{2}+L_{2} / 2\right)\right)$ be satisfied. Let $u$ be the solution of (1.1) - (1.3). Then for sufficiently large $\bar{\sigma}$, the UPML problem (4.6) - (4.7) has a unique solution $\hat{u}$. Moreover, there exists a constant $C$ depending only on $\gamma_{0}, k_{2} / k_{1}, L_{2} / L_{1}$ but independent of $k_{j}, L_{j}$, and $d_{j}, j=1,2$, such that

$$
\|u-\hat{u}\|_{H^{1}\left(\Omega_{1}\right)} \leq C\left(1+\hat{C}^{-1}\right) \gamma_{1}\left(1+k_{1} L_{1}\right)^{3} \alpha_{m}^{3}\left(1+\frac{\bar{\sigma}}{L_{1}}\right)^{2} e^{-k_{1} \gamma_{0} \bar{\sigma}}\|\hat{u}\|_{H^{1 / 2}\left(\Gamma_{1}\right)}
$$

Proof. We prove the estimate (7.8) first. Suppose the solution $\hat{u}$ of (4.6) - (4.7) exists. By (3.18) and (7.3), simple integration by parts implies

$$
a(u-\hat{u}, \varphi)=\hat{a}(\hat{u}, \varphi)-a(\hat{u}, \varphi)=\langle T \hat{u}-\hat{T} \hat{u}, \varphi\rangle_{\Gamma_{1}}, \quad \forall \varphi \in H^{1}\left(\Omega_{\mathrm{PML}}\right) .
$$

Using (3.19) and Lemma 7.1, we obtain (7.8).

Now we turn to the well-posedness of the UPML problem. By the Fredholm alternative theorem we only need to show the uniqueness of the UPML problem (4.6) 
- (4.7). For that purpose we assume (4.6) - (4.7) has a solution $\hat{u}$ for $f=0$ in (4.6) and $g=0$ in (4.7). By the uniqueness of the scattering problem we know that the corresponding scattering solution $u=0$ in $\Omega_{1}$. Thus (7.8) implies

$$
\|\hat{u}\|_{H^{1}\left(\Omega_{1}\right)} \leq C\left(1+\hat{C}^{-1}\right) \gamma_{1}\left(1+k_{1} L_{1}\right)^{3} \alpha_{m}^{3}\left(1+\frac{\bar{\sigma}}{L_{1}}\right)^{2} e^{-k_{1} \gamma_{0} \bar{\sigma}}\|\hat{u}\|_{H^{1 / 2}\left(\Gamma_{1}\right)} .
$$

Thus for sufficiently large $\bar{\sigma}$ we conclude that $\hat{u}=0$ on $\Omega_{1}$. That $\hat{u}$ also vanishes in $\Omega_{2}$ follows from Lemma 5.1 since $\hat{u}$ satisfies the PML equation in the PML layer with homogeneous boundary conditions.

8. Concluding remarks. In this paper we have proved that the solution of the UPML problem converges exponentially to the solution of the Helmholtz scattering problem in two-layer media. The convergence can be realized by either enlarging the thickness of the PML layer or enlarging the PML absorbing coefficients. The proof is based the method of complex coordinate stretching and a new representation of the Green function which is essential for the estimate for the modified Green function. We will extend the results of this paper to design an adaptive UPML method and report the numerical examples in a forthcoming paper.

Acknowledgement. The authors are very grateful to the referees of the paper for their constructive comments that improve the paper.

\section{REFERENCES}

[1] R.A. Adams, Sobolev Spaces, Academic Press, New-York, 1978.

[2] J. P. BÉRÉNGER, A perfectly matched layer for the absorption of electromagnetic waves, J. Comput. Physics, 114 (1994), pp. 185-200.

[3] J. Chen and Z. Chen, An adaptive perfectly matched layer technique for 3-D time-harmonic electromagnetic scattering problems, Math. Comp., 77 (2008), pp. 673-698.

[4] Z. Chen AND H. Wu, An adaptive finite element method with perfectly matched absorbing layers for the wave scattering by periodic structures, SIAM J. Numer. Anal., 41(2003), pp. 799-826.

[5] Z. Chen And X. LiU, An adaptive perfectly matched layer technique for time-harmonic scattering problems, SIAM J. Numer. Anal., 43(2005), pp. 645-671.

[6] Z. Chen AND X. WU, An adaptive uniaxial perfectly matched layer method for time-harmonic scattering problems, Numerical Mathematics: Theory, Methods and Applications, 1 (2008), pp. 113-137.

[7] Z. Chen, T. Cui and L. Zhang, An adaptive uniaxial perfectly matched layer method for time harmonic Maxwell scattering problems, to appear.

[8] W. C. Chew And W. WeEdon, A 3D perfectly matched medium from modified Maxwell's equations with stretched coordinates, Microwave Opt. Tech. Lett., 7 (1994), pp. 599-604.

[9] W.-C. Chew, Waves and Fields in Inhomogenous Media, Van Nodtrand Reimhold, New York, 1990.

[10] F. Collino And P. Monk, The perfectly matched layer in curvilinear coordinates, SIAM J. Sci. Comput., 19 (1998), pp. 2061-2090.

[11] D. Colton and R. Kress, Integral Equation Methods in Scattering Theory, John Wiley \& Sons, New York, 1983.

[12] J. Coyle And P. Monk, The Finite Element Approximation of Scattering in a Layered Medium, in Analytical and Computational Methods in Scattering and Applied Mathematics, F. Santosa and I. Stakgold, Eds., Chapman \& Hall/CRC, London. Research Notes in Mathematics number 417 (2000). pp. 67-84.

[13] T. Hagstrom, Radiation boundary conditions for the numerical simulation of waves, Acta Numerica (1999), 47-106.

[14] T. Hohage, F. Schmidt, And L. Zschiedrich, Solving time-harmonic scattering problems based on the pole condition. II: Convergence of the PML method, SIAM J. Math. Anal., 35 (2003), pp. 547-560. 
[15] G. Kristensson, A uniqueness theorem for Helmholtz equation: penetrable media with an infinite interface, SIAM J. Math. Anal., 11(1980), pp. 1104-1116.

[16] M. Lassas And E. Somersalo, On the existence and convergence of the solution of PML equations, Computing, 60 (1998), pp. 229-241.

[17] M. Lassas and E. Somersalo, Analysis of the PML equations in general convex geometry, Proceeding of the Royal Society of Edinburg (2001), pp. 1183 - 1207.

[18] P. Monk, Finite Element Methods for Maxwells Equations, Oxford University Press, 2003.

[19] J.C. NÉdÉLEC, Acoustic and Electromagnetic Equations, Integral Representations for Harmonic Problems, Springer-Verlag, New York, 2001.

[20] F.M. ODEH, Uniqueness theorems for the Helmholtz equation in domains with infinite boundaries, J. Math. Mech., 12 (1963), pp.857-868.

[21] M. Paulus, P. Gay-Balmas and O.J.F. Martin, Accurate and efficient computation of Green's tensor for stratified media, Physical Review E, 62 (2000), pp. 5797-5807.

[22] G.F. RoACH AND B. ZHANG, The limiting-amplitude principle for the wave propagation problem with two unbounded media, Math. Proc. Camb. Phil. Soc., 112 (1992), pp. 207-223.

[23] G.F. Roach And B. Zhang, A transmission problem for the reduced wave equation in inhomogeneous media with an infinite interface, Proc. Royal Soc. London A, 436 (1992), pp. 121-140.

[24] F.L. Teixeira And W.C. Chew, Advances in the theory of perfectly matched layers, in: W.C. Chew et al, (eds.), Fast and Efficient Algorithms in Computational Electromagnetics, pp. 283- 346, Artech House, Boston, 2001.

[25] E. Turkel And A. Yefet, Absorbing PML boundary layers for wave-like equations, Appl. Numer. Math., 27 (1998), pp. 533-557. 